\title{
THERMO-VISCO-ELASTICITY WITH RATE-INDEPENDENT PLASTICITY IN ISOTROPIC MATERIALS UNDERGOING THERMAL EXPANSION*
}

\author{
SÖREN BARTELS ${ }^{1}$ AND TOMÁŠ ROUBÍČEK ${ }^{2,3}$
}

\begin{abstract}
We consider a viscoelastic solid in Kelvin-Voigt rheology exhibiting also plasticity with hardening and coupled with heat-transfer through dissipative heat production by viscoplastic effects and through thermal expansion and corresponding adiabatic effects. Numerical discretization of the thermodynamically consistent model is proposed by implicit time discretization, suitable regularization, and finite elements in space. Fine a-priori estimates are derived, and convergence is proved by careful successive limit passage. Computational 3D simulations illustrate an implementation of the method as well as physical effects of residual stresses substantially depending on rate of heat treatment.
\end{abstract}

Mathematics Subject Classification. 35K85, 49S05, 65M60, 74C05, 80A17.

Received November 30, 2009. Revised June 17, 2010.

Published online October 11, 2010.

\section{INTRODUCTION}

Thermal expansion in metallic bodies may create enormous elastic stresses if the temperature profile varies considerably. It occurs both within manufacturing processes (especially heat treatment of large bulks) and sometimes in working regimes, too. These "thermo-elastic" stresses may trigger activated inelastic processes, typically slip plasticity or even damage. Here we focus on plasticity and consider also hardening effects. Mechanical energy dissipated during the plastic deformation is converted to changes of an internal structure of the material due to hardening but also to heat, which ultimately couples the mechanical and heat parts. Moreover, thermal expansion leads to heat production/consumption due to adiabatic effects.

\footnotetext{
Keywords and phrases. Thermodynamics of plasticity, Kelvin-Voigt rheology, hardening, thermal expansion, adiabatic effects, finite element method, implicit time discretization, convergence.

* The authors warmly thank Professor Alexander Mielke and Dr. Jiř̌ Plešek for many fruitful discussions, and an anonymous referee for many helpful comments on an earlier version of the manuscript. S.B. acknowledges support by "Nečas center for mathematical modeling" LC 06052 (MS̆MT C̆R). T.R. acknowledges the hospitality of SFB 611 "Singular phenomena and scaling in mathematical models" of the University of Bonn, as well as partial support from the grants A 100750802

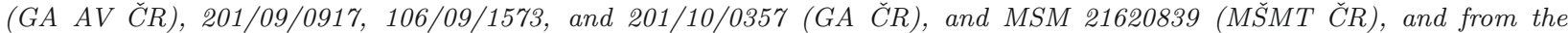

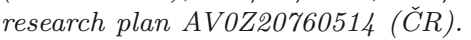

${ }^{1}$ Institut für Numerische Simulation, Rheinische Friedrich-Wilhelms-Universität Bonn, Wegelerstraße 6, 53115 Bonn, Germany. bartels@ins .uni.bonn.de

2 Mathematical Institute, Charles University, Sokolovská 83, 18675 Praha 8, Czech Republic.

${ }^{3}$ Institute of Thermomechanics of the ASCR, Dolejškova 5, 18200 Praha 8, Czech Republic.
} 
There is an extensive engineering literature addressing thermoplasticity in thermally expanding materials, employing computationally sophisticated models, e.g. $[1,7,9,19,26,27]$, sometimes even at large strains $[10,16$, $20,32,33]$ but lacking a rigorous mathematical justification.

Mathematically supported theories seem, however, nearly missing for a long time. This was mainly because the relevant $L^{1}$-theory for the heat equation was developed only in the 1990s [4-6], and the mathematical theory for rate-independent processes is even more recent, cf. [13,15,21-24], as well as the interpolation technique of the adiabatic-heat term in three-dimensional case [29], and the coupling with rate-independent processes with viscous/inertial effects [30] and thermal effects [31].

The main mathematical difficulties are related to finite strain and multiplicative plasticity at finite strains, in evolution driven even by mere elastic response if kinetic effects are counted, and coupling of rate-independent processes with rate-dependent ones. In fact, each of the above mentioned three difficulties represents itself a hard open problem, especially in a three-dimensional setting and if no regularization (e.g. by capillarity or higher viscosity is involved). This is why we adopt the following simplifications: small strains and strain-driven linearized additive plasticity, and a linear viscoelastic response. On the other hand, we allow for a fully rateindependent plastic flow rule although, of course, the whole system is necessarily rate dependent due to the heat transfer, and here also due to considered kinetic and viscous effects. As mentioned above, we also consider hardening to avoid spatial concentration of plastic strains as has been studied in the isothermal case in [13] which, in general, would lead to awkward interactions of concentrating plastic-strain rate with thermal effects. Recently, rigorous mathematical studies for thermoviscoplasticity at small strains had been performed in [3] (considering, however, a rate-dependent plastic flow rule and no thermal expansion effects) and, as mentioned above, in [31] (considering general analytical scheme for a slightly different class of generalized standard materials with gradient theories for internal parameters but without numerical analysis and the modification for the linearized non-gradient plasticity only outlined in [31], Rem. 4.5 with Ex. 5.1).

The model will be formulated in Section 2 where also its thermodynamics will be exposed. We confine ourselves to trace-free plastic strain and to isotropic materials as far as both elastic response and thermal expansion are concerned. This implies a mathematically important cancellation effect owing to the fact that the thermal-expansion strain is diagonal and thus orthogonal to the "plastic stress", $c f .(2.24)$ below. Although this restricts generality and excludes e.g. single-crystal plasticity which is remarkably anisotropic, important applications in engineering which standardly treat polycrystalline (and thus isotropic) metals are allowed by the presented theory.

The main purpose of this paper, performed in Sections 3 and 4, is to develop an implementable numerical scheme for this model and prove its stability, i.e. a-priori estimates, and also convergence to a suitably defined weak solution of the model. In Section 5, the efficiency of the proposed numerical strategy is demonstrated on a 3D example. We illustrate it on a physically motivated example, exhibiting rate-dependent effects of thermal coupling producing residual elastic stresses and plastic deformation depending on rate of heat treatment of a steel workpiece.

\section{THE MODEL WITHIN THERMODYNAMICS}

We consider a bounded Lipschitz domain $\Omega \subset \mathbb{R}^{d}, d \leq 3$. The state variables will be the displacement $u: \Omega \rightarrow \mathbb{R}^{d}$, the plastic strain $\pi: \Omega \rightarrow \mathbb{R}_{\mathrm{dev}}^{d \times d}$, possibly a scalar isotropic hardening parameter $\eta$, and the temperature $\theta: \Omega \rightarrow \mathbb{R}$, where

$$
\mathbb{R}_{\mathrm{dev}}^{d \times d}:=\left\{A \in \mathbb{R}_{\mathrm{sym}}^{d \times d} ; \operatorname{tr}(A)=0\right\} \quad \text { and } \quad \mathbb{R}_{\mathrm{sym}}^{d \times d}:=\left\{A \in \mathbb{R}^{d \times d} ; A^{\top}=A\right\} .
$$

The variables $(\pi, \eta)$ play the role of internal parameters. We consider plastic response determined by a convex closed neighbourhood of the origin, say $K \subset \mathbb{R}_{\mathrm{sym}}^{d \times d} \times \mathbb{R}$, defining an elasticity domain, while its boundary is called the yield surface and has the meaning of the stress that triggers the evolution of plastic strains; we refer to Section 5 for a specific example. Let $\delta_{K}$ denote its indicator function and $\delta_{K}^{*}$ the Fenchel-Legendre conjugate functional to $\delta_{K}$ with respect to the inner product $\sigma: e=\sum_{i, j=1}^{d} \sigma_{i j} e_{i j}$. Note that the physical dimension 
of $\sigma: e$ is $P a=\mathrm{J} \cdot \mathrm{m}^{-3}$ so that $K$ determining the degree-1 positively homogeneous "plastic" dissipation potential $\delta_{K}^{*}$, acting on the dimensionless tensor $\pi$ and on the dimensionless internal hardening variable $\eta$, has indeed the dimension $\mathrm{J} \cdot \mathrm{m}^{-3}$. For a simpler notation, we write

$$
\zeta_{1}(\dot{\pi}, \dot{\eta}):=\delta_{K}^{*}(\dot{\pi}, \dot{\eta})
$$

We remark that the condition $0 \in \operatorname{int}(K)$ implies that $\zeta_{1}=\delta_{K}^{*}$ is coercive. The set $K$ must be unbounded. More specifically, we assume that

$$
K=K_{0} \oplus K_{1}, \quad \text { where } K_{0} \subset \mathbb{R}_{\mathrm{dev}}^{d \times d} \times \mathbb{R} \text { is convex, } K_{1} \text { is the orthogonal complement of } \mathbb{R}_{\mathrm{dev}}^{d \times d} \times \mathbb{R} .
$$

This implies that $\zeta_{1}$ is finite only on $\mathbb{R}_{\mathrm{dev}}^{d \times d} \times \mathbb{R}$ and that $\pi(t, \cdot) \in \mathbb{R}_{\mathrm{dev}}^{d \times d}$ a.e. in $\Omega$ provided that $\pi_{0}(\cdot) \in \mathbb{R}_{\mathrm{dev}}^{d \times d}$ a.e. in $\Omega$. Therefore, no deviator operator occurs in (2.5) below. We remark that, alternatively, one may consider a (bounded) set $K_{0} \subset \mathbb{R}_{\mathrm{dev}}^{d \times d}$ and involve the deviator operator in (2.5) and (2.10b) below; then $\zeta_{1}$ would be real-valued.

Considering a Kelvin-Voigt-type viscous material, our model will consist of the equilibrium equation balancing inertial, viscous, and elastic mechanical forces,

$$
\varrho \frac{\partial^{2} u}{\partial t^{2}}-\operatorname{div}\left(\mathbb{D} \frac{\partial e(u)}{\partial t}\right)-\operatorname{div}(\mathbb{C}(e(u)-\pi-\mathbb{E} \theta))=0,
$$

where $\varrho$ is the mass density, $\mathbb{D}$ the tensor determining the viscous-type response, $\mathbb{C}$ the tensor determining the elastic response, and $\mathbb{E}$ the thermal expansion tensor, while the evolution of the internal parameters $\pi$ and $\eta$ are governed by the inclusion

$$
\partial \zeta_{1}\left(\frac{\partial \pi}{\partial t}, \frac{\partial \eta}{\partial t}\right)+\left(\begin{array}{c}
\mathbb{C} \pi+\mathbb{H} \pi \\
b \eta
\end{array}\right) \ni\left(\begin{array}{c}
\mathbb{C} e(u) \\
0
\end{array}\right)
$$

where $b>0$ is an isotropic hardening coefficient and $\mathbb{H}$ is a symmetric positive semidefinite fourth-order tensor determining the kinematic hardening, and the heat transfer/production is governed by the equation

$$
c_{\mathrm{v}}(\theta) \frac{\partial \theta}{\partial t}-\operatorname{div}(\mathbb{K}(\theta) \nabla \theta)=\zeta_{1}\left(\frac{\partial \pi}{\partial t}, \frac{\partial \eta}{\partial t}\right)+2 \zeta_{2}\left(\frac{\partial e(u)}{\partial t}\right)-\theta \mathbb{E}: \mathbb{C} \frac{\partial e(u)}{\partial t}
$$

where $c_{\mathrm{v}}=c_{\mathrm{v}}(\theta)$ is the heat capacity and $\mathbb{K}=\mathbb{K}(\theta)$ is the thermal conductivity tensor,

$$
\zeta_{2}(\dot{e})=\frac{1}{2} \mathbb{D} \dot{e}: \dot{e}
$$

is the pseudopotential of viscous-dissipative forces, ":" denotes the product of two $(d \times d)$-tensors, and $e(u)$ is the small-strain tensor defined as

$$
e_{i j}(u)=\frac{1}{2}\left(\frac{\partial u_{i}}{\partial x_{j}}+\frac{\partial u_{j}}{\partial x_{i}}\right)
$$

Throughout this paper, we assume isotropic thermal expansion, i.e.

$$
\mathbb{E}=\alpha \mathbb{I}
$$

with $\alpha$ a single thermal-expansion coefficient. 
Using the identity $\left[\partial \zeta_{1}\right]^{-1}=\partial \zeta_{1}^{*}$ and taking into account (2.9), the equation and inclusion (2.4)-(2.5) can equivalently be written in a form which is more standard in engineering literature, namely

$$
\begin{aligned}
& \varrho \frac{\partial^{2} u}{\partial t^{2}}=\operatorname{div} \sigma-\alpha \nabla \theta \quad \text { with } \quad \sigma=\sigma_{\text {visc }}+\sigma_{\text {elast }}, \quad \sigma_{\text {visc }}=\mathbb{D} \frac{\partial e(u)}{\partial t}, \quad \sigma_{\text {elast }}=\mathbb{C}(e(u)-\pi), \\
& \left(\begin{array}{c}
\partial \pi / \partial t \\
\partial \eta / \partial t
\end{array}\right) \in \partial \zeta_{1}^{*}\left(\begin{array}{c}
-\sigma_{\text {plast }} \\
-b \eta
\end{array}\right) \quad \text { with } \quad \sigma_{\text {plast }}=-\sigma_{\text {elast }}+\mathbb{H} \pi,
\end{aligned}
$$

which reveals the total stress $\sigma$ to be composed from the viscous and the elastic parts $\sigma_{\text {visc }}$ and $\sigma_{\text {elast }}$ (which expresses just the Kelvin-Voigt rheology), and the plastic stress $\sigma_{\text {plast }}$ involving $\mathbb{H} \pi$ is in the position of the back stress to the elastic stress $\sigma_{\text {elast }}$. This is also known as Ziegler's type model [34]. Alternatively, $\sigma_{\text {plast }}$ would have involved only the deviatoric part of $\sigma_{\text {elast }}$ and $\mathbb{H}$ would have had to be assumed as $\mathbb{R}_{\mathrm{dev}}^{d \times d} \rightarrow \mathbb{R}_{\mathrm{dev}}^{d \times d}$ if the mentioned variant of $\zeta_{1}$ valued in $\mathbb{R}$ were considered.

The above equations/inclusion (2.4)-(2.8) are to hold on the space/time domain $Q:=(0, T) \times \Omega$ with $T>0$ a fixed time horizon.

As we focus on processes in the bulk, we consider only the simplest boundary conditions, namely a prescribed normal stress and heat flux on $\Gamma:=\partial \Omega$ :

$$
\begin{array}{lr}
\left(\mathbb{D} \frac{\partial e(u)}{\partial t}+\mathbb{C}(e(u)-\pi-\mathbb{E} \theta)\right) \nu=g & \text { on } \Gamma, \\
(\mathbb{K}(\theta) \nabla \theta) \cdot \nu=f & \text { on } \Gamma,
\end{array}
$$

where "." denotes the scalar product of two vectors and $\nu$ is the outward normal to $\Gamma$.

The energetics of the model is based on the mechanical part of the internal energy

$$
\Phi(u, \pi, \eta):=\frac{1}{2} \int_{\Omega} \mathbb{C}(e(u)-\pi):(e(u)-\pi)+\mathbb{H} \pi: \pi+b \eta^{2} \mathrm{~d} x,
$$

the kinetic energy

$$
T_{\text {kin }}(\dot{u}):=\frac{1}{2} \int_{\Omega} \varrho|\dot{u}|^{2} \mathrm{~d} x,
$$

the dissipation energy rate

$$
\Xi(\dot{u}, \dot{\pi}, \dot{\eta}):=\int_{\Omega} \zeta_{1}(\dot{\pi}, \dot{\eta})+2 \zeta_{2}(e(\dot{u})) \mathrm{d} x
$$

with $\zeta_{1}$ from (2.2) and $\zeta_{2}$ from (2.7), the thermal part of the internal energy

$$
E(\theta):=\int_{\Omega} h(\theta) \mathrm{d} x, \quad \text { where } \quad h(\theta):=\int_{0}^{\theta} c_{\mathrm{v}}(w) \mathrm{d} w,
$$

and the power of external mechanical forces and heating

$$
F(t, \dot{u})=\int_{\Gamma} g(t, x) \cdot \dot{u}(x)+f(t, x) \mathrm{d} S .
$$

The energetics of the model (2.4)-(2.6) can be obtained by testing (2.4)-(2.6) respectively by the velocity $\frac{\partial u}{\partial t}$, by the plastic strain rate $\frac{\partial \pi}{\partial t}$, and by 1 , which gives after using Green's formula for both (2.4) and (2.6) together 
with the boundary conditions (2.11) and eventually by summation the energy balance

$$
\frac{\mathrm{d}}{\mathrm{d} t}\left(T_{\operatorname{kin}}\left(\frac{\partial u}{\partial t}\right)+\Phi(u, \pi, \eta)+E(\theta)\right)=F\left(t, \frac{\partial u}{\partial t}\right) ;
$$

cf. (3.7d) below.

We consider an initial-boundary-value problem for the system (2.4)-(2.8). Hence, we take the initial conditions

$$
u(0, \cdot)=u_{0}, \quad \frac{\partial u}{\partial t}(0, \cdot)=\dot{u}_{0}, \quad \pi(0, \cdot)=\pi_{0}, \quad \eta(0, \cdot)=\eta_{0}, \quad \theta(0, \cdot)=\theta_{0} .
$$

Also, the non-negativity of temperature is ensured provided $\theta_{0} \geq 0$ and provided the boundary heat flux $f$ is non-negative, $c f$. also the proof of Proposition 4.4 below.

Throughout the article, we will rely on the following data qualification. The positive definite fourth order tensors $\mathbb{C}=\left[\mathbb{C}_{i j k l}\right], \mathbb{D}=\left[\mathbb{D}_{i j k l}\right]$ are assumed to be symmetric and isotropic so that in fact

$$
\begin{array}{rlrl}
\mathbb{C}_{i j k l}=\lambda_{\mathrm{e}} \delta_{i j} \delta_{k l}+\mu_{\mathrm{e}}\left(\delta_{i k} \delta_{j l}+\delta_{i l} \delta_{j k}\right), & \mathbb{D}_{i j k l}=\lambda_{\mathrm{v}} \delta_{i j} \delta_{k l}+\mu_{\mathrm{v}}\left(\delta_{i k} \delta_{j l}+\delta_{i l} \delta_{j k}\right), \\
\text { with } \mu_{\mathrm{e}}, \mu_{\mathrm{v}}>0, & \lambda_{\mathrm{e}}>-\frac{2}{d} \mu_{\mathrm{e}}, & \lambda_{\mathrm{v}}>-\frac{2}{d} \mu_{\mathrm{v}},
\end{array}
$$

with $\delta$ denoting here the Kronecker symbol, $\lambda$ 's and $\mu$ 's are the Lamé constants. Thus the elastic stress is $\mathbb{C} e=$ $\lambda_{\mathrm{e}} \operatorname{tr}(e) \mathbb{I}+2 \mu_{\mathrm{e}} e$ with $\mathbb{I}=\left[\delta_{i j}\right]$ denoting the unit matrix, and corresponding energy is $\frac{1}{2} \mathbb{C} e: e=\frac{1}{2} \lambda_{\mathrm{e}}|\operatorname{tr}(e)|^{2}+\mu_{\mathrm{e}}|e|^{2}$ and, as a quadratic form of $e$, it is positive definite, and similarly also the quadratic form $e \mapsto \frac{1}{2} \mathbb{D} e: e$ is positive definite. In fact, for the analysis presented below, we need the isotropy only for $\mathbb{C}$ but it would be physically inconsistent to have $\mathbb{D}$ anisotropic.

One can derive thermodynamics of the above model by postulating the Helmholtz free energy as

$$
\psi(e, \pi, \eta, \theta)=\frac{1}{2} \mathbb{C}(e-\pi-\mathbb{E} \theta):(e-\pi-\mathbb{E} \theta)+\frac{1}{2} \mathbb{H} \pi: \pi+\frac{b}{2} \eta^{2}-\frac{\theta^{2}}{2} \mathbb{C E}: \mathbb{E}-\phi_{0}(\theta) .
$$

Then entropy is given by

$$
s=s(e, \theta):=-\frac{\partial}{\partial \theta} \psi=\phi_{0}^{\prime}(\theta)-\mathbb{E}: \mathbb{C} e .
$$

Note that the thermo-mechanically coupling terms in (2.20) are linear in terms of $\theta$, so that the mechanical variables separate from temperature in (2.21) and thus $c_{\mathrm{v}}=c_{\mathrm{v}}(e, \theta)=\theta \frac{\partial}{\partial \theta} s(e, \theta)=\theta \phi_{0}^{\prime \prime}(\theta)$ in (2.6) does not depend on these mechanical variables, which facilitates the analysis of the heat equation considerably.

The equation (2.6) itself can be derived from the entropy equation

$$
\theta \frac{\partial s}{\partial t}-\operatorname{div}(\mathbb{K} \nabla \theta)=\xi \quad \text { with the dissipation rate } \xi:=\zeta_{1}\left(\frac{\partial \pi}{\partial t}, \frac{\partial \eta}{\partial t}\right)+2 \zeta_{2}\left(\frac{\partial e(u)}{\partial t}\right),
$$

which takes, in general, in the form

$$
\theta \psi_{\theta \theta}^{\prime \prime}(\theta) \frac{\partial \theta}{\partial t}-\operatorname{div}(\mathbb{K}(\theta) \nabla \theta)=\xi-\theta \psi_{\theta e}^{\prime \prime}(e, \pi, \eta, \theta): \frac{\partial e(u)}{\partial t}-\theta \psi_{\theta \pi}^{\prime \prime}(e, \pi, \eta, \theta): \frac{\partial \pi}{\partial t}-\theta \psi_{\theta \eta}^{\prime \prime}(e, \pi, \eta, \theta) \frac{\partial \eta}{\partial t} .
$$

Note that $\int_{\Omega} \xi \mathrm{d} x=\Xi\left(\frac{\partial u}{\partial t}, \frac{\partial \pi}{\partial t}, \frac{\partial \eta}{\partial t}\right)$ with $\Xi$ from (2.14). Note also that, as $b$ is independent of $\theta$, the last term in (2.23) vanishes. Moreover, the important facts are that also $\mathbb{H}$ is independent of $\theta$ and that, owing to (2.3) together with (2.9) and (2.19) we have the orthogonality

$$
\mathbb{C} \pi: \mathbb{E}=\alpha\left(\lambda_{\mathrm{e}} \operatorname{tr}(\pi) \mathbb{I}+2 \mu_{\mathrm{e}} \pi\right): \mathbb{I}=\alpha\left(d \lambda_{\mathrm{e}}+2 \mu_{\mathrm{e}}\right) \operatorname{tr}(\pi)=0
$$


provided also $\operatorname{tr}\left(\pi_{0}\right)=0$. This guarantees that the term $\theta \psi_{\theta \pi}^{\prime \prime}(e, \pi, \eta, \theta): \frac{\partial \pi}{\partial t}=\theta \mathbb{E}: \mathbb{C} \frac{\partial \pi}{\partial t}$ in $(2.23)$ vanishes. This facilitates the analysis in our rate-independent plasticity substantially, because otherwise an $L^{1}$-type term $\frac{\partial \pi}{\partial t}$ would be in product with temperature $\theta$ which hardly can be "compact" in an $L^{\infty}$-space.

At least formally, assuming positivity of temperature and $f \geq 0$, and realizing that always $\xi \geq 0$, from (2.22) we can see the Clausius-Duhem inequality

$$
\frac{\mathrm{d}}{\mathrm{d} t} \int_{\Omega} s \mathrm{~d} x=\int_{\Omega} \operatorname{div}\left(\mathbb{K} \frac{\nabla \theta}{\theta}\right)+\frac{\mathbb{K} \nabla \theta \cdot \nabla \theta}{\theta^{2}}+\frac{\xi}{\theta} \mathrm{d} x=\int_{\Omega} \frac{\mathbb{K} \nabla \theta \cdot \nabla \theta}{\theta^{2}}+\frac{\xi}{\theta} \mathrm{d} x+\int_{\Gamma} \frac{f}{\theta} \mathrm{d} S \geq 0 ;
$$

obviously, $\mathbb{K} \nabla \theta \cdot \nabla \theta / \theta^{2}+\xi / \theta$ is the entropy-production rate. Note also that a combination of (2.9), (2.19), and (2.24) allows us to write $\psi$ from (2.20) in the more specific form

$$
\psi(e, \pi, \eta, \theta)=\frac{\lambda_{\mathrm{e}}}{2}|\operatorname{tr}(e-\pi)|^{2}+\mu_{\mathrm{e}}|e-\pi|^{2}-\alpha\left(d \lambda_{\mathrm{e}}+2 \mu_{\mathrm{e}}\right) \theta \operatorname{tr}(e)+\frac{1}{2} \mathbb{H} \pi: \pi+\frac{b}{2} \eta^{2}-\phi_{0}(\theta)
$$

where (2.24) was used, too. In fact, (2.25) in the form $\frac{\mathrm{d}}{\mathrm{d} t} \int_{\Omega} s \mathrm{~d} x \geq \int_{\Omega} \frac{\mathbb{K} \nabla \theta \cdot \nabla \theta}{\theta^{2}}+\frac{\xi}{\theta} \mathrm{d} x+\int_{\Gamma} \frac{f}{\theta} \mathrm{d} S$ can conversely serve as the origin of the constitutional relation for the entropy $s=-\frac{\partial}{\partial \theta} \psi, c f .(2.21)$, and the elastic stress $\sigma=\frac{\partial}{\partial e} \psi, c f .(2.10 \mathrm{~b})$, as well as the driving force for the flow rule $\frac{\partial}{\partial(\pi, \eta)} \psi=(\mathbb{H} \pi-\sigma, b \zeta)$.

\section{ENTHALPY TRANSFORMATION AND WEAK FORMULATION}

It is desirable to allow for a certain growth of $c_{\mathrm{v}}(\cdot)$ if we have the viscosity in the form $\mathbb{D} e\left(\frac{\partial u}{\partial t}\right)$ in order to be able to treat the adiabatic term, $c f$. [29]. On the other hand, the technique from [29] specifically relies on Galerkin's method and does not seem directly transferable if also the time discretization is involved, which is in turn needed both for designing a fully discrete scheme and for efficient treatment of the rate-independent flow rule. The particular difficulty is in establishing the limit of a time-discretization of the nonlinear term $c_{\mathrm{v}}(\theta) \frac{\partial \theta}{\partial t}$. Therefore, we first write the original system (2.4)-(2.6) in terms of enthalpy instead of temperature, using so-called enthalpy transformation

$$
w=h_{0}(\theta):=\int_{0}^{\theta} c_{\mathrm{v}}(r) \mathrm{d} r
$$

thus $h_{0}$ is a primitive function to $c_{\mathrm{v}}$ normalized such that $h_{0}(0)=0$. Further, we define

$$
\Theta(w):=\left\{\begin{array}{ll}
h_{0}^{-1}(w) & \text { if } w \geq 0, \\
0 & \text { if } w<0,
\end{array} \quad \mathcal{K}(w):=\frac{\mathbb{K}(\Theta(w))}{c_{\mathrm{v}}(\Theta(w))}\right.
$$

where $h_{0}^{-1}$ here denotes the inverse function to $h_{0}$. This transforms the system (2.4)-(2.6) into the form

$$
\begin{aligned}
& \varrho \frac{\partial^{2} u}{\partial t^{2}}-\operatorname{div}\left(\mathbb{D} e\left(\frac{\partial u}{\partial t}\right)+\mathbb{C}(e(u)-\pi-\Theta(w) \mathbb{E})\right)=0, \\
& \partial \zeta_{1}\left(\frac{\partial \pi}{\partial t}, \frac{\partial \eta}{\partial t}\right)+\left(\begin{array}{c}
\mathbb{C} \pi+\mathbb{H} \pi \\
b \eta
\end{array}\right) \ni\left(\begin{array}{c}
\mathbb{C} e(u) \\
0
\end{array}\right), \\
& \frac{\partial w}{\partial t}-\operatorname{div}(\mathcal{K}(w) \nabla w)=\zeta_{1}\left(\frac{\partial \pi}{\partial t}, \frac{\partial \eta}{\partial t}\right)+2 \zeta_{2}\left(\frac{\partial e(u)}{\partial t}\right)+\Theta(w) \mathbb{E}: \mathbb{C} e\left(\frac{\partial u}{\partial t}\right) .
\end{aligned}
$$


We will call (3.3c) shortly the enthalpy equation rather than the heat-transfer equation in the enthalpy formulation. The boundary conditions (2.11) transforms to

$$
\begin{array}{ll}
\left(\mathbb{D} e\left(\frac{\partial u}{\partial t}\right)+\mathbb{C}(e(u)-\pi-\mathbb{E} \Theta(w))\right) \nu=g & \text { on } \Gamma \\
(\mathcal{K}(w) \nabla w) \cdot \nu=f & \text { on } \Gamma
\end{array}
$$

while the initial conditions (2.18) transform into

$$
u(0, \cdot)=u_{0}, \quad \frac{\partial u}{\partial t}(0, \cdot)=\dot{u}_{0}, \quad \pi(0, \cdot)=\pi_{0}, \quad \eta(0, \cdot)=\eta_{0}, \quad w(0, \cdot)=h_{0}\left(\theta_{0}\right)
$$

The following definition of a certain sort of a weak solution has been devised in [31], based on the concept of socalled energetic solution invented by Mielke et al. $[15,21,23,24]$ for the theory of rate independent processes and adapted also for coupling with viscous/inertial effects in [30]. We refer to [31], Proposition 3.2, for justification (and not entirely obvious fact) that this definition is indeed selective in the sense that, under an additional absolute continuity of $\frac{\partial \pi}{\partial t}$ and $\frac{\partial \eta}{\partial t}$, it gives indeed a conventional notion of a weak solution. For an isothermal situation, $c f$. also [30], Proposition 5.2. It should be however emphasized that this additional regularity of $\frac{\partial \pi}{\partial t}$ and $\frac{\partial \eta}{\partial t}$ hardly can be expected due to the fully rate-independent flow rule, which just makes the devised concept properly fitted with this problem.

We consider an evolution in the time interval $I:=(0, T)$ with a fixed time horizon $T>0$ and denote $Q:=(0, T) \times \Omega, \Sigma:=(0, T) \times \partial \Omega$, and $\bar{I}:=[0, T]$. We will use a standard notation for function spaces, namely the space of the continuous $\mathbb{R}^{k}$-valued functions $C\left(\bar{\Omega} ; \mathbb{R}^{k}\right)$, its dual $\mathcal{M}\left(\bar{\Omega} ; \mathbb{R}^{k}\right)$ (i.e., up to an isometric isomorphism, the space of Borel measures), the continuously differentiable functions $C^{1}\left(\bar{\Omega} ; \mathbb{R}^{k}\right)$, the Lebesgue space $L^{p}\left(\Omega ; \mathbb{R}^{k}\right)$, the Sobolev space $W^{1, p}\left(\Omega ; \mathbb{R}^{k}\right)$, and the Bochner space of $X$-valued Bochner measurable $p$-integrable functions $L^{p}(I ; X)$. If $X=\left(X^{\prime}\right)^{*}$, the notation $L_{\mathrm{w} *}^{\infty}(I ; X)$ stands for space of weakly* measurable functions $I \rightarrow X$; this space is dual to the space $L^{1}\left(I ; X^{\prime}\right)$ and, in general, is not equal to $L^{\infty}(I ; X)$. If $X$ is separable reflexive, then $L^{\infty}(I ; X)=L_{\mathrm{w} *}^{\infty}(I ; X)$ by Pettis' theorem, however. Moreover, we denote by $B(\bar{I} ; X)$, $B_{\mathrm{w} *}(\bar{I} ; X), \mathrm{BV}(\bar{I} ; X)$ or $C_{\mathrm{w}}(\bar{I} ; X)$ the Banach space of functions $\bar{I} \rightarrow X$ that are bounded Bochner measurable, bounded weakly* measurable, have a bounded variation or are weakly continuous, respectively; note that all these functions are defined everywhere on $\bar{I}$. We will use the notation $q^{\prime}=q /(q-1)$ for the conjugate exponent to $q$. Instead of $u(t, \cdot)$ or $\pi(t, \cdot)$ or $\eta(t, \cdot)$ or $w(t, \cdot)$, we will write briefly $u(t)$ or $\pi(t)$ or $\eta(t)$ or $w(t)$, respectively.

Definition 3.1 (energetic solution). Assuming (2.19)-(3.10), we call a quadruple $(u, \pi, \eta, w)$ with

$$
\begin{aligned}
& u \in C_{\mathrm{w}}\left(I ; W^{1,2}\left(\Omega ; \mathbb{R}^{d}\right)\right) \\
& \frac{\partial u}{\partial t} \in L^{2}\left(I ; W^{1,2}\left(\Omega ; \mathbb{R}^{d}\right)\right) \cap W^{1,2}\left(I ; W^{1,2}\left(\Omega ; \mathbb{R}^{d}\right)^{*}\right), \\
& \pi \in B\left(\bar{I} ; L^{2}\left(\Omega ; \mathbb{R}_{\mathrm{dev}}^{d \times d}\right)\right) \cap \operatorname{BV}\left(\bar{I} ; L^{1}\left(\Omega ; \mathbb{R}_{\mathrm{dev}}^{d \times d}\right)\right), \\
& \eta \in B\left(\bar{I} ; L^{2}(\Omega)\right) \cap \operatorname{BV}\left(\bar{I} ; L^{1}(\Omega)\right), \\
& w \in L^{r}\left(I ; W^{1, r}(\Omega)\right) \cap L^{\infty}\left(I ; L^{1}(\Omega)\right) \cap B_{\mathrm{w} *}(\bar{I} ; \mathcal{M}(\bar{\Omega})) \quad \text { with any } 1 \leq r<\frac{d+2}{d+1}, \\
& \frac{\partial w}{\partial t} \in \mathcal{M}\left(\bar{I} ; W^{1+d, 2}(\Omega)^{*}\right)
\end{aligned}
$$


an energetic solution to (3.3) with the initial/boundary conditions (3.5) and (4.3) if the following five conditions hold:

(i) The weakly formulated momentum-equilibrium equation (3.3a) with (4.3a), (4.3b) holds, i.e. for all $v \in C^{1}\left(\bar{Q} ; \mathbb{R}^{d}\right)$ such that $\left.v\right|_{\Sigma_{0}}=0$,

$$
\begin{aligned}
& \int_{\Omega} \varrho \frac{\partial u}{\partial t}(T) \cdot v(T) \mathrm{d} x+\int_{Q}\left(\mathbb{D} e\left(\frac{\partial u}{\partial t}\right)+\mathbb{C}(e(u)-\pi-\mathbb{E} \Theta(w))\right): e(v)-\varrho \frac{\partial u}{\partial t} \cdot \frac{\partial v}{\partial t} \mathrm{~d} x \mathrm{~d} t \\
& =\int_{\Sigma} g \cdot v \mathrm{~d} S \mathrm{~d} t+\int_{\Omega} \varrho \dot{u}_{0} \cdot v(0) \mathrm{d} x .
\end{aligned}
$$

(ii) The weakly formulated enthalpy equation (3.3c) with (4.3c) holds, i.e. for all $v \in C^{1}(\bar{Q})$ with $v(T)=0$,

$$
\begin{array}{r}
\int_{Q} \mathcal{K}(w) \nabla w \cdot \nabla v-w \frac{\partial v}{\partial t}-\Theta(w) \mathbb{E}: \mathbb{C} e\left(\frac{\partial u}{\partial t}\right) v-\mathbb{D} e\left(\frac{\partial u}{\partial t}\right): e\left(\frac{\partial u}{\partial t}\right) v \mathrm{~d} x \mathrm{~d} t \\
=\int_{\bar{Q}} v \mathfrak{h}_{\pi, \eta}(\mathrm{d} x \mathrm{~d} t)+\int_{\Omega} w_{0} v(0) \mathrm{d} x+\int_{\Sigma} f v \mathrm{~d} S \mathrm{~d} t
\end{array}
$$

where $w_{0}=h_{0}\left(\theta_{0}\right)$ and $\mathfrak{h}_{\pi, \eta}$ is a measure (= heat produced by rate-independent dissipation) defined by prescribing its values for every closed set of the type $A:=\left[t_{1}, t_{2}\right] \times B$ with $B$ a Borel subset of $\bar{\Omega}$ by

$$
\begin{aligned}
\mathfrak{h}_{\pi, \eta}(A):= & \operatorname{Var}_{\zeta_{1}}\left(\left.(\pi, \eta)\right|_{B} ; t_{1}, t_{2}\right) \quad \text { with } \\
& \operatorname{Var}_{\zeta_{1}}\left(z ; t_{1}, t_{2}\right):=\sup \sum_{i=1}^{k} \int_{\Omega} \zeta_{1}\left(z\left(s_{i}, x\right)-z\left(s_{i-1}, x\right)\right) \mathrm{d} x
\end{aligned}
$$

where the supremum is taken over all partitions of the type $t_{1} \leq s_{0}<\ldots<s_{k} \leq t_{2}, k \in \mathbb{N}$.

(iii) The total energy equality holds, i.e. with $\Phi$ and $T_{\text {kin }}$ from (2.12) and (2.13),

$$
\begin{aligned}
T_{\text {kin }}\left(\frac{\partial u}{\partial t}(T)\right)+\Phi & (u(T), \pi(T), \eta(T))+\int_{\bar{\Omega}} w(T, \mathrm{~d} x) \\
& =T_{\text {kin }}\left(\dot{u}_{0}\right)+\Phi\left(u_{0}, \pi_{0}, \eta_{0}\right)+\int_{\Omega} h_{0}\left(\theta_{0}\right) \mathrm{d} x+\int_{\Sigma} g \cdot \frac{\partial u}{\partial t}+f \mathrm{~d} S \mathrm{~d} t .
\end{aligned}
$$

(iv) The "semistability" holds for any $\tilde{\pi} \in L^{2}\left(\Omega ; \mathbb{R}_{\operatorname{dev}}^{d \times d}\right)$ and $\tilde{\eta} \in L^{2}(\Omega)$ and for all $t \in[0, T]$, i.e.

$$
\Phi(u(t), \pi(t), \eta(t)) \leq \Phi(u(t), \tilde{\pi}, \tilde{\eta})+\int_{\Omega} \zeta_{1}(\tilde{\pi}-\pi(t), \tilde{\eta}-\eta(t)) \mathrm{d} x .
$$

(v) The initial conditions $u(0)=u_{0}, \pi(0)=\pi_{0}$, and $\eta(0)=\eta_{0}$ hold.

Note also that (3.6f) makes values of $w(t)$ well defined in the sense of $W^{1+n, 2}(\Omega)^{*}$ and (3.6e) further shows that even $w(t) \in \mathcal{M}(\bar{\Omega})$, which has been exploited in (3.7d) for the time $t=T$. It should be emphasized that $t \mapsto w(t)$ cannot be expected to be continuous in any sense because, since $\zeta_{1}$ is homogeneous degree-1, the measure $\mathfrak{h}_{\pi, \eta}$ may concentrate at particular time instances.

In addition to (2.19) which guarantees that $\mathbb{C}$ and $\mathbb{D}$ are positive definite, we will assume throughout this article that

$$
\varrho \geq 0 .
$$


Other assumptions are on nonlinearities $c_{\mathrm{v}}$ and $\mathbb{K}$, namely we assume:

$$
\begin{aligned}
& c_{\mathrm{V}}:[0,+\infty) \rightarrow \mathbb{R}^{+} \text {continuous, } \\
& \exists \omega_{1} \geq \omega \geq 1, c_{1} \geq c_{0}>0 \forall \theta \in \mathbb{R}^{+}: \quad c_{0}(1+\theta)^{\omega-1} \leq c_{\mathrm{v}}(\theta) \leq c_{1}(1+\theta)^{\omega_{1}-1}, \\
& \mathcal{K}: \mathbb{R} \rightarrow \mathbb{R}^{d \times d} \text { bounded, continuous, and } \inf _{(w, \xi) \in \mathbb{R}^{\prime} \times \mathbb{R}^{d},|\xi|=1} \mathcal{K}(w) \xi \cdot \xi>0 \quad \text { with } \mathcal{K} \text { from (3.2) below; }
\end{aligned}
$$

later in (4.26) we impose further restrictions on $\omega$. As far as the loading qualification concerns, we assume

$$
\begin{aligned}
& g \in L^{2}\left(I ; L^{q}\left(\Gamma ; \mathbb{R}^{d}\right)\right), \quad q \geq 2-2 / d \quad(\text { or } q>1 \text { if } d \leq 2) \\
& f \in L^{1}(\Sigma), \quad f \geq 0, \\
& u_{0} \in W^{1,2}\left(\Omega ; \mathbb{R}^{d}\right) \\
& \dot{u}_{0} \in L^{2}\left(\Omega ; \mathbb{R}^{d}\right) \\
& \pi_{0} \in L^{2}\left(\Omega ; \mathbb{R}_{\mathrm{dev}}^{d \times d}\right) \\
& \eta_{0} \in L^{2}(\Omega), \quad \eta_{0}>0 \\
& \theta_{0} \in L^{\omega}(\Omega), \quad \theta_{0} \geq 0
\end{aligned}
$$

where we denoted $\Sigma:=I \times \Gamma$ in $(3.10 \mathrm{~b})$.

\section{Discretization AND NUMERical ANALYsis}

To prove stability of any discrete scheme needed for possible convergence, one must inevitably deal with executing fine a-priori estimates. This is not entirely easy in coupled systems with super-linear growth of nonmonotone terms, as it is typically the case of thermodynamically consistent continuum-mechanical problems. It has to be done essentially in two steps: first the physical internal and kinetic energy is to be estimated uniformly in time using (and proving) also non-negativity of the enthalpy, and, from this, some additional finer estimates by using Gagliardo-Nirenberg interpolation several times. In this second step, one estimates especially the gradient of enthalpy and also total dissipated energy, cf. also [29] and, for the plasticity, especially [31]. Further important phenomenon here is that, proving existence of a solution, we need to pass to the limit in the non-linear Nemytskiı operators induced by the dissipation heat $\xi$. Another peculiarity is that, due to degree- 1 homogeneity of $\zeta_{1}$, the heat equation has its right-hand side not only in $L^{1}(Q)$ (as it would be in case of higher-degree homogeneity of dissipative-force potential) but even in measures. For this, the key trick is to recover the exact energy balance in the limit. In addition to [31], there are some further peculiarities related with spatial discretisation. In particular, it seems difficult to make spatial discretization of the term $-\operatorname{div}(\mathcal{K}(w) \nabla w)$ compatible with the maximum principle even on acute triangulations if $\mathcal{K}$ is nonconstant (and the qualification $(3.9 \mathrm{~b}),(3.9 \mathrm{~d})$ with $(4.26)$ below exclude constant $\mathcal{K}$ if $d \geq 2)$.

Therefore, a design of a convergent numerical scheme is technically rather delicate. Following [31], we will use a fully implicit time-discretization with a constant time-step $\tau>0$, assuming $K_{\tau}=T / \tau \in \mathbb{N}$ and defining the backward difference operator by

$$
\mathrm{D}_{t} \phi^{k}:=\frac{\phi^{k}-\phi^{k-1}}{\tau}
$$

for any sequence $\left\{\phi^{k}\right\}_{k \geq 0}$, combined with a regularization of the momentum equation and of the flow rule, (using as a parameter just the time-step $\tau>0$ ) and of the enthalpy equation (using a parameter $\varepsilon=\varepsilon(\tau)>0$ whose dependence on $\tau$ will be implicitly specified later in (4.34)). More specifically, we consider the following 
recursive increment formula

$$
\begin{aligned}
& \varrho \mathrm{D}_{t}^{2} u_{\tau}^{k}-\operatorname{div}\left(\mathbb{D} e\left(\mathrm{D}_{t} u_{\tau}^{k}\right)+\mathbb{C}\left(e\left(u_{\tau}^{k}\right)-\pi_{\tau}^{k}-\mathbb{E} \Theta\left(w_{\tau}^{k}\right)\right)+\tau\left|e\left(u_{\tau}^{k}\right)\right|^{\gamma-2} e\left(u_{\tau}^{k}\right)\right)=0 \\
& \partial \zeta_{1}\left(\mathrm{D}_{t} \pi_{\tau}^{k}, \mathrm{D}_{t} \eta_{\tau}^{k}\right)+\left(\begin{array}{c}
\mathbb{C} \pi_{\tau}^{k}+\mathbb{H} \pi_{\tau}^{k} \\
b \eta_{\tau}^{k}
\end{array}\right)+\tau \mathcal{S}\left(\begin{array}{c}
\pi_{\tau}^{k} \\
\eta_{\tau}^{k}
\end{array}\right) \ni\left(\begin{array}{c}
\mathbb{C} e\left(u_{\tau}^{k}\right) \\
0
\end{array}\right) \\
& \mathrm{D}_{t} w_{\tau}^{k}-\operatorname{div}\left(\mathcal{K}\left(w_{\tau}^{k}\right) \nabla w_{\tau}^{k}\right)+\varepsilon(\tau)\left|w_{\tau}^{k}\right|^{\beta-2} w_{\tau}^{k}=\zeta_{1}\left(\mathrm{D}_{t} \pi_{\tau}^{k}, \mathrm{D}_{t} \eta_{\tau}^{k}\right)+\mathbb{D} e\left(\mathrm{D}_{t} u_{\tau}^{k}\right): e\left(\mathrm{D}_{t} u_{\tau}^{k}\right)+\Theta\left(w_{\tau}^{k}\right) \mathbb{E}: \mathbb{C} e\left(\mathrm{D}_{t} u_{\tau}^{k}\right)
\end{aligned}
$$

for $k=1, \ldots, K_{\tau}=T / \tau$ with the corresponding boundary conditions

$$
\begin{array}{r}
\left(\mathbb{D} e\left(\mathrm{D}_{t} u_{\tau}^{k}\right)+\mathbb{C}\left(e\left(u_{\tau}^{k}\right)-\pi_{\tau}^{k}-\mathbb{E} \Theta\left(w_{\tau}^{k}\right)\right)+\tau\left|e\left(u_{\tau}^{k}\right)\right|^{\gamma-2} e\left(u_{\tau}^{k}\right)\right) \nu=g_{\tau}^{k} \\
\left(\mathcal{K}\left(w_{\tau}^{k}\right) \nabla w_{\tau}^{k}\right) \cdot \nu=f_{\tau}^{k}
\end{array}
$$

on $\Gamma$, starting for $k=1$ by using

$$
u_{\tau}^{0}=u_{0, \tau}, \quad u_{\tau}^{-1}=u_{0, \tau}-\tau \dot{u}_{0}, \quad \pi_{\tau}^{0}=\pi_{0, \tau}, \quad \eta_{\tau}^{0}=\eta_{0, \tau}, \quad w_{\tau}^{0}=w_{0, \tau}
$$

where

$$
g_{\tau}^{k}(t, x):=\frac{1}{\tau} \int_{(k-1) \tau}^{k \tau} g(t, x) \mathrm{d} t \text { and } f_{\tau}^{k}:=\frac{1}{\tau} \int_{(k-1) \tau}^{k \tau} \tilde{f}_{\tau}(t, x) \mathrm{d} t
$$

Note that, in (4.4) and (4.5), we regularized the initial values and the boundary flux $u_{0}, z_{0}, w_{0}$, and $f$ by $u_{0, \tau}, z_{0, \tau}, w_{0, \tau}$, and $\tilde{f}_{\tau}$, respectively, $c f .(4.8)$ below. Moreover, $\mathcal{S}$ in $(4.2 \mathrm{~b})$ is a regularizing selfadjoint positive definite linear operator having the quadratic potential

$$
\frac{1}{2}|z|_{W^{a, 2}(\Omega)}^{2} \text { with some } 0<a<1 / 2
$$

applied component-wise in $(4.2 \mathrm{~b})$, with $|\cdot|_{W^{a, 2}(\Omega)}$ meaning the standard seminorm in the Sobolev-Slobodetskiu fractional-derivative space. Later we can also use its square root $\mathcal{S}^{1 / 2}$, defined as a selfadjoint positive definite operator such that $\mathcal{S}^{1 / 2} \circ \mathcal{S}^{1 / 2}=\mathcal{S}$. Thus

$$
\mathcal{S}: W^{a, 2}(\Omega) \rightarrow W^{a, 2}(\Omega)^{*} \quad \text { and } \quad \mathcal{S}^{1 / 2}: W^{a, 2}(\Omega) \rightarrow L^{2}(\Omega)
$$

Note that we regularized also the initial state for the mechanical part (but not the initial velocity).

Let us comment the purpose of the regularizing terms. The " $\gamma$-term" in $(4.2 \mathrm{a})$ and the " $\beta$-term" in $(4.2 \mathrm{c})$ are to compensate the superlinear growth of the right-hand-side terms in the heat equation; the former one has already been used in [31] for a mere time discretization, while the latter one is here needed because, in the spatially discretized scheme, we will not be able to test by nonlinear functions of $w$, in contrast with the spatially continuous case in [31]. Eventually, the "S $\mathcal{S}$-term" in (4.2b) helps to make a limit passage in space discretization without using numerical integration formulae, $c f .(4.20)$ below. 
As far as the (regularized) initial and boundary conditions and the loading concerns, we assume

$$
\begin{array}{lll}
u_{0, \tau} \in W^{1, \gamma}\left(\Omega ; \mathbb{R}^{d}\right), & \lim _{\tau \downarrow 0} \sqrt[\gamma]{\tau}\left\|e\left(u_{0, \tau}\right)\right\|_{L^{\gamma}\left(\Omega ; \mathbb{R}^{d \times d}\right)}=0, & \lim _{\tau \downarrow 0} u_{0, \tau}=u_{0} \text { in } W^{1,2}\left(\Omega ; \mathbb{R}^{d}\right), \\
\pi_{0, \tau} \in W^{a, 2}\left(\Omega ; \mathbb{R}^{d \times d+1}\right), & \lim _{\tau \downarrow 0} \sqrt{\tau}\left\|\pi_{0, \tau}\right\|_{W^{a, 2}\left(\Omega ; \mathbb{R}^{d \times d+1}\right)}=0, & \lim _{\tau \downarrow 0} \pi_{0, \tau}=\pi_{0} \text { in } L^{2}\left(\Omega ; \mathbb{R}^{d \times d}\right), \\
\eta_{0, \tau} \in W^{a, 2}\left(\Omega ; \mathbb{R}^{d \times d+1}\right), & \lim _{\tau \downarrow 0} \sqrt{\tau}\left\|\eta_{0, \tau}\right\|_{W^{a, 2}\left(\Omega ; \mathbb{R}^{d \times d+1}\right)}=0, & \lim _{\tau \downarrow 0} \eta_{0, \tau}=\eta_{0} \text { in } L^{2}(\Omega), \\
w_{0, \tau} \in L^{2}(\Omega), & \lim _{\tau \downarrow 0} \sqrt{\tau}\left\|w_{0, \tau}\right\|_{L^{2}(\Omega)}=0, & \lim _{\tau \downarrow 0} w_{0, \tau}=w_{0}:=h_{0}\left(\theta_{0}\right) \text { in } L^{1}(\Omega), \\
\tilde{f}_{\tau} \in L^{\infty}(\Sigma), \quad \tilde{f}_{\tau} \geq 0, & \lim _{\tau \downarrow 0} \sqrt{\tau}\left\|\tilde{f}_{\tau}\right\|_{L^{2}\left(I ; L^{4 / 3}(\Gamma)\right)}=0, & \lim _{\tau \downarrow 0} \tilde{f}_{\tau}=f \text { in } L^{1}(\Sigma) .
\end{array}
$$

We will further make a spatial discretization. For this, we assume that we are given a sequence of triangulations $\left\{\mathcal{T}_{h}\right\}_{h>0}$ of the polyhedral domain $\Omega$ without hanging nodes but otherwise entirely general. We suppose that $h>0$ range over countable sets of positive real numbers with accumulation points at 0 , and that $\max _{E \in \mathcal{T}_{h}} \operatorname{diam}(E) \leq h$.

We consider $C^{0}$-conforming P1-elements for the approximation of $u$ and $w$ and P0-elements for the approximation of $\pi$ and $\eta$. The finite-dimensional subspaces of $L^{2}(\Omega)$ and $W^{1,2}(\Omega)$ related to P0- and P1-elements and subordinate to the triangulation $\mathcal{T}_{h}$ respectively by $V_{0, h}$ and $V_{1, h}$.

For $j=0,1$, the $L^{2}$ orthogonal projection onto $V_{j, h}$ is denoted by $P_{j, h}$. We have the following approximation property at our disposal for any $1 \leq \gamma<\infty$ :

$$
\begin{array}{lll}
\forall v \in L^{2}(\Omega): & P_{0, h} v \rightarrow v & \text { in } L^{2}(\Omega), \\
\forall v \in W^{1, \gamma}(\Omega): & P_{1, h} v \rightarrow v & \text { in } W^{1, \gamma}(\Omega),
\end{array}
$$

as follows from approximation results in [8].

Then we devise the Galerkin scheme as follows. We seek $\left(u_{\tau h}^{k}, \pi_{\tau h}^{k}, \eta_{\tau h}^{k}, w_{\tau h}^{k}\right) \in V_{1, h}^{d} \times V_{0, h}^{d \times d} \times V_{0, h} \times V_{1, h}$, with $\pi_{\tau h}^{k} \in \mathbb{R}_{\text {dev }}^{d \times d}$ a.e. on $\Omega$, satisfying

$$
\begin{gathered}
\int_{\Omega} \varrho \mathrm{D}_{t}^{2} u_{\tau h}^{k} \cdot v+\left(\mathbb{D} e\left(\mathrm{D}_{t} u_{\tau h}^{k}\right)+\mathbb{C}\left(e\left(u_{\tau h}^{k}\right)-\pi_{\tau h}^{k}-\mathbb{E} \Theta\left(w_{\tau h}^{k}\right)\right)+\tau\left|e\left(u_{\tau h}^{k}\right)\right|^{\gamma-2} e\left(u_{\tau h}^{k}\right)\right): e(v) \mathrm{d} x \\
=\int_{\Gamma} g_{\tau}^{k} \cdot v \mathrm{~d} S \quad \text { for all } v \in V_{1, h}^{d}, \\
\int_{\Omega} \zeta_{1}(\tilde{\pi}, \tilde{\eta})+\left(\mathbb{C} \pi_{\tau h}^{k}-\mathbb{C} e\left(u_{\tau h}^{k}\right)+\mathbb{H} \pi_{\tau h}^{k}\right):\left(\tilde{\pi}-\mathrm{D}_{t} \pi_{\tau h}^{k}\right)+b \eta_{\tau h}^{k}\left(\tilde{\eta}-\mathrm{D}_{t} \eta_{\tau h}^{k}\right)+\tau \mathcal{S}^{1 / 2} \pi_{\tau h}^{k}: \mathcal{S}^{1 / 2}\left(\tilde{\pi}-\mathrm{D}_{t} \pi_{\tau h}^{k}\right) \\
\quad+\tau \mathcal{S}^{1 / 2} \eta_{\tau h}^{k} \mathcal{S}^{1 / 2}\left(\tilde{\eta}-\mathrm{D}_{t} \eta_{\tau h}^{k}\right) \mathrm{d} x \geq \int_{\Omega} \zeta_{1}\left(\mathrm{D}_{t} \pi_{\tau h}^{k}, \mathrm{D}_{t} \eta_{\tau h}^{k}\right) \mathrm{d} x \quad \text { for all }(\tilde{\pi}, \tilde{\eta}) \in V_{1, h}^{d \times d} \times V_{1, h}, \\
\int_{\Omega}\left(\mathrm{D}_{t} w_{\tau h}^{k}+\varepsilon(\tau)\left|w_{\tau h}^{k}\right|^{\beta-2} w_{\tau h}^{k}\right) v+\mathcal{K}\left(w_{\tau h}^{k}\right) \nabla w_{\tau h}^{k} \cdot \nabla v-\zeta_{1}\left(\mathrm{D}_{t} \pi_{\tau h}^{k}, \mathrm{D}_{t} \eta_{\tau h}^{k}\right) v \\
\quad-\mathbb{D} e\left(\mathrm{D}_{t} u_{\tau h}^{k}\right): e\left(\mathrm{D}_{t} u_{\tau h}^{k}\right) v \mathrm{~d} x=\int_{\Omega} \Theta\left(w_{\tau h}^{k}\right) \mathbb{E}: \mathbb{C} e\left(\mathrm{D}_{t} u_{\tau h}^{k}\right) v \mathrm{~d} x+\int_{\Gamma} f_{\tau, h}^{k} v \mathrm{~d} S \quad \text { for all } v \in V_{1, h} .
\end{gathered}
$$

Let us define the piecewise affine interpolant $\left(u_{\tau h}, \pi_{\tau h}, \eta_{\tau h}, w_{\tau h}\right)$ by

$$
\begin{array}{r}
{\left[u_{\tau h}, \pi_{\tau h}, \eta_{\tau h}, w_{\tau h}\right](t):=\frac{t-(k-1) \tau}{\tau}\left(u_{\tau h}^{k}, \pi_{\tau h}^{k}, \eta_{\tau h}^{k}, w_{\tau h}^{k}\right)+\frac{k \tau-t}{\tau}\left(u_{\tau h}^{k-1}, \pi_{\tau h}^{k-1}, \eta_{\tau h}^{k-1}, w_{\tau h}^{k-1}\right)} \\
\text { for } t \in[(k-1) \tau, k \tau] \text { with } k=0, \ldots, K_{\tau}:=T / \tau .
\end{array}
$$


Besides, we define also the back-ward piecewise constant interpolant $\left(\bar{u}_{\tau h}, \bar{\pi}_{\tau h}, \bar{\eta}_{\tau h}, \bar{w}_{\tau h}\right)$ by

$$
\left[\bar{u}_{\tau h}, \bar{\pi}_{\tau h}, \bar{\eta}_{\tau h}, \bar{w}_{\tau h}\right](t):=\left(u_{\tau h}^{k}, \pi_{\tau h}^{k}, \eta_{\tau h}^{k}, w_{\tau h}^{k}\right) \quad \text { for } \quad(k-1) \tau<t \leq k \tau, k=1, \ldots, K_{\tau}
$$

Similarly, we will later use $u_{\tau}, \bar{u}_{\tau}$, etc. We will also use the notation $\bar{g}_{\tau}$ and $\bar{f}_{\tau}$ defined by $\left.\bar{g}_{\tau}\right|_{((k-1) \tau, k \tau]}=g_{\tau}^{k}$ and $\left.\bar{f}_{\tau}\right|_{((k-1) \tau, k \tau]}=f_{\tau}^{k}$ for $k=1, \ldots, K_{\tau}$.

Lemma 4.1 (existence and estimates of discrete solutions). Let (2.19), (3.9), (3.10), and (4.8) hold. Moreover, let

$$
\beta>2, \quad \gamma>\max \left(4, \frac{2 \omega}{\omega-1}\right), \quad \text { and } \quad \omega>1
$$

Then there exists a solution $\left(u_{\tau h}^{k}, \pi_{\tau h}^{k}, \eta_{\tau h}^{k}, w_{\tau h}^{k}\right) \in V_{1, h}^{d} \times V_{0, h}^{d \times d} \times V_{0, h} \times V_{1, h}$, with $\pi(\cdot) \in \mathbb{R}_{\mathrm{dev}}^{d \times d}$ a.e. on $\Omega$, for the system (4.10). Moreover,

$$
\begin{aligned}
& \left\|u_{\tau h}\right\|_{W^{1, \infty}\left(I ; W^{1, \gamma}\left(\Omega ; \mathbb{R}^{d}\right)\right)} \leq C_{\tau}, \\
& \left\|\pi_{\tau h}\right\|_{W^{1, \infty}\left(I ; W^{a, 2}\left(\Omega ; \mathbb{R}_{\mathrm{dev}}^{d \times d}\right)\right)} \leq C_{\tau}, \\
& \left\|\eta_{\tau h}^{k}\right\|_{W^{1, \infty}\left(I ; W^{a, 2}(\Omega)\right)} \leq C_{\tau}, \\
& \left\|w_{\tau h}^{k}\right\|_{W^{1, \infty}\left(I ; W^{1,2}(\Omega)\right)} \leq C_{\tau},
\end{aligned}
$$

with some $C_{\tau}$ independent of $h$ and with $a \in(0,1 / 2)$ referring to (4.6).

Sketch of the proof. We can see existence of a solution to (4.10) by a standard argument for coercive pseudomonotone set-valued operators; cf. e.g. [17] for a general concept or, here, [28], Section 5.3, for inclusions with pseudomonotone operators whose set-valued part has a convex potential. The coercivity of the underlying operator can be shown by testing (4.10a)-(4.10c) by $u_{\tau h}^{k} \in V_{1, h}^{d}, \pi_{\tau h}^{k} \in V_{0, h}^{d \times d}, \eta_{\tau h}^{k} \in V_{0, h}$, and $w_{\tau h}^{k} \in V_{1, h}$, respectively. Note that these test-functions live in the corresponding finite-dimensional spaces and are thus legal for this test. It is important that the right-hand sides of (4.10a), (4.10c) have the growth that can be dominated by the growth of the coercive terms in the left-hand sides; this is ensured by having taken $\beta$ and $\gamma$ large enough and by the assumption $(3.9 \mathrm{~b})$ which ensures a sublinear growth of $\Theta$, namely

$$
\Theta(w) \leq\left(\frac{w}{\omega c_{0}}+1\right)^{1 / \omega}-1 \leq\left(\frac{w}{\omega c_{0}}\right)^{1 / \omega}
$$

because obviously $h_{0}(\theta) \geq \omega c_{0}(1+\theta)^{\omega}-\omega c_{0}, c f$. the definition (3.2). Realize that the coercivity can be estimated (up to multiplicative constants) as $|e|^{\gamma}+|\pi|^{2}+|\eta|^{2}+|w|^{\beta}$ which indeed dominates the growth of the "righthand-side terms" is of the type $|w|^{1 / \omega}|e|+(|\pi|+|\eta|)|w|+|e|^{2}|w|+|w|^{1+1 / \omega}|e|$. The term $(|\pi|+|\eta|)|w|$ bears the estimation by $\delta|\pi|^{2}+\delta|\eta|^{2}+\delta|w|^{\beta}+C_{\delta}$ and similarly $|e|^{2}|w| \leq \delta|e|^{\gamma}+\delta|w|^{2}+C_{\delta}$ with any $\delta>0$ and some $C_{\delta}$; here $\beta>2$ and $\gamma>4$ have respectively been used. The last term can be estimated as $|w|^{1+1 / \omega}|e| \leq$ $\frac{1}{\gamma}|e|^{\gamma}+|w|^{(1+1 / \omega) \gamma /(\gamma-1)} \leq \frac{1}{\gamma}|e|^{\gamma}+\frac{1}{2}|w|^{2}+C_{\gamma}$ for some $C_{\gamma} \in \mathbb{R}$; here the condition $\gamma>2 \omega /(\omega-1)$ has originated.

The a-priori estimates (4.14) then follows from the above test by standard procedure, i.e. by using the Hölder, the Young, and the discrete Gronwall inequalities.

Lemma 4.2 (convergence for $h \downarrow 0)$. There is a subsequence of $\left\{\left(u_{\tau h}, \pi_{\tau h}, \eta_{\tau h}, w_{\tau h}\right)\right\}_{h>0}$ converging for $h \downarrow 0$ weakly* in the topologies indicated in (4.14) to some $\left(u_{\tau}, \pi_{\tau}, \eta_{\tau}, w_{\tau}\right)$ and each quadruple obtained by such way 
is a weak solution to (4.2)-(4.3), i.e. in term of the interpolants

$$
\begin{aligned}
& \varrho \mathrm{D}_{t}^{2} u_{\tau}-\operatorname{div}\left(\mathbb{D} e\left(\frac{\partial u_{\tau}}{\partial t}\right)+\mathbb{C}\left(e\left(\bar{u}_{\tau}\right)-\bar{\pi}_{\tau}-\mathbb{E} \Theta\left(\bar{w}_{\tau}\right)\right)+\tau\left|e\left(\bar{u}_{\tau}\right)\right|^{\gamma-2} e\left(\bar{u}_{\tau}\right)\right)=0 \\
& \partial \zeta_{1}\left(\frac{\partial \pi_{\tau}}{\partial t}, \frac{\partial \eta_{\tau}}{\partial t}\right)+\left(\begin{array}{c}
\mathbb{C} \bar{\pi}_{\tau}+\mathbb{H} \bar{\pi}_{\tau} \\
b \bar{\eta}_{\tau}
\end{array}\right)+\tau \mathcal{S}\left(\begin{array}{c}
\bar{\pi}_{\tau} \\
\bar{\eta}_{\tau}
\end{array}\right) \ni\left(\begin{array}{c}
\mathbb{C} e\left(\bar{u}_{\tau}\right) \\
0
\end{array}\right) \\
& \frac{\partial w_{\tau}}{\partial t}-\operatorname{div}\left(\mathcal{K}\left(\bar{w}_{\tau}\right) \nabla \bar{w}_{\tau}\right)+\varepsilon(\tau)\left|\bar{w}_{\tau}\right|^{\beta-2} \bar{w}_{\tau}=\zeta_{1}\left(\frac{\partial \pi_{\tau}}{\partial t}, \frac{\partial \eta_{\tau}}{\partial t}\right)+\mathbb{D} e\left(\frac{\partial u_{\tau}}{\partial t}\right): e\left(\frac{\partial u_{\tau}}{\partial t}\right)+\Theta\left(\bar{w}_{\tau}\right) \mathbb{E}: \mathbb{C} e\left(\frac{\partial u_{\tau}}{\partial t}\right)
\end{aligned}
$$

with the boundary conditions

$$
\begin{array}{r}
\left(\mathbb{D} e\left(\frac{\partial u_{\tau}}{\partial t}\right)+\mathbb{C}\left(e\left(\bar{u}_{\tau}\right)-\bar{\pi}_{\tau}-\mathbb{E} \Theta\left(\bar{w}_{\tau}\right)\right)+\tau\left|e\left(\bar{u}_{\tau}\right)\right|^{\gamma-2} e\left(\bar{u}_{\tau}\right)\right) \nu=\bar{g}_{\tau}, \\
\left(\mathcal{K}\left(\bar{w}_{\tau}\right) \nabla \bar{w}_{\tau}\right) \cdot \nu=\bar{f}_{\tau},
\end{array}
$$

and with the initial conditions (4.4); of course, $\mathrm{D}_{t}^{2} u_{\tau}$ in (4.16a) means the piece-wise constant interpolant in time and $\left(\bar{u}_{\tau}, \bar{\pi}_{\tau}, \bar{\eta}_{\tau}, \bar{w}_{\tau}\right)$ is the limit of a subsequence of $\left\{\left(\bar{u}_{\tau h}, \bar{\pi}_{\tau h}, \bar{\eta}_{\tau h}, \bar{w}_{\tau h}\right)\right\}_{h>0}$ and simultaneously also the piece-wise constant interpolant in time corresponding to $\left(u_{\tau}, \pi_{\tau}, \eta_{\tau}, w_{\tau}\right)$.

Sketch of the proof. By Banach's selection principle, we first select a weakly* convergent subsequence. Due to the construction of $V_{1, h}$, we have the approximation property $(4.9 \mathrm{~b})$ at our disposal. Hence we can consider also a sequence $\left\{\widetilde{u}_{\tau h}\right\}_{h>0}$ converging strongly to $u_{\tau}$ even in $W^{1, \infty}\left(I ; W^{1, \gamma}\left(\Omega ; \mathbb{R}^{d}\right)\right)$ and such that $\widetilde{u}_{\tau h}: I \rightarrow V_{1, h}^{d}$; here one must take into account that $\tau>0$ is fixed hence only a finite number of values of $u_{\tau}$ is to be approximated by using (4.9b).

Due to the dissipative-heat term in (4.16c), we need to prove the strong convergence $\frac{\partial}{\partial t} e\left(u_{\tau h}\right) \rightarrow \frac{\partial}{\partial t} e\left(u_{\tau}\right)$ in $L^{2}\left(Q ; \mathbb{R}^{d \times d}\right)$. To this goal, we first use the so-called $d$-monotonicity of $e \mapsto \mathbb{C} e+|e|^{\gamma-2} e$ to prove the strong convergence $e\left(\bar{u}_{\tau h}\right) \rightarrow e\left(\bar{u}_{\tau}\right)$ in $L^{\gamma}\left(Q ; \mathbb{R}^{d \times d}\right)$ by the estimate

$$
\begin{aligned}
& \tau\left(\left\|e\left(\bar{u}_{\tau h}\right)\right\|_{L^{\gamma}\left(Q ; \mathbb{R}^{d \times d}\right)}^{\gamma-1}-\left\|e\left(\bar{u}_{\tau}\right)\right\|_{L^{\gamma}\left(Q ; \mathbb{R}^{d \times d}\right)}^{\gamma-1}\right)\left(\left\|e\left(\bar{u}_{\tau h}\right)\right\|_{L^{\gamma}\left(Q ; \mathbb{R}^{d \times d}\right)}-\left\|e\left(\bar{u}_{\tau}\right)\right\|_{L^{\gamma}\left(Q ; \mathbb{R}^{d \times d}\right)}\right) \\
& \leq \int_{Q} \mathbb{C} e\left(\bar{u}_{\tau h}-\bar{u}_{\tau}\right): e\left(\bar{u}_{\tau h}-\bar{u}_{\tau}\right)+\tau\left(\left|e\left(\bar{u}_{\tau h}\right)\right|^{\gamma-2} e\left(\bar{u}_{\tau h}\right)-\left|e\left(\bar{u}_{\tau}\right)\right|^{\gamma-2} e\left(\bar{u}_{\tau}\right)\right): e\left(\bar{u}_{\tau h}-\bar{u}_{\tau}\right) \mathrm{d} x \mathrm{~d} t \\
&+\int_{\Omega} \frac{1}{2} \mathbb{D} e\left(u_{\tau h}(T)-u_{\tau}(T)\right): e\left(u_{\tau h}(T)-u_{\tau}(T)\right) \mathrm{d} x \\
& \leq \int_{Q} \mathbb{C}\left(\bar{\pi}_{\tau h}+\Theta\left(\bar{w}_{\tau h}\right) \mathbb{E}\right): e\left(\bar{u}_{\tau h}-\widetilde{u}_{\tau h}\right)-\varrho D_{t}^{2} u_{\tau h} \cdot\left(\bar{u}_{\tau h}-\widetilde{u}_{\tau h}\right) \\
&-\left(\mathbb{C} e\left(\bar{u}_{\tau}\right)+\tau\left|e\left(\bar{u}_{\tau}\right)\right|^{\gamma-2} e\left(\bar{u}_{\tau}\right)+\mathbb{D} e\left(\frac{\partial u_{\tau}}{\partial t}\right)\right): e\left(\bar{u}_{\tau h}-\widetilde{u}_{\tau h}\right) \mathrm{d} x \mathrm{~d} t+\int_{\Sigma} \bar{g}_{\tau} \cdot\left(\bar{u}_{\tau h}-\widetilde{u}_{\tau h}\right) \mathrm{d} S \mathrm{~d} t \\
&+\int_{Q} \mathbb{C} e\left(\bar{u}_{\tau h}-\bar{u}_{\tau}\right): e\left(\widetilde{u}_{\tau h}-\bar{u}_{\tau}\right)+\tau\left(\left|e\left(\bar{u}_{\tau h}\right)\right|^{\gamma-2} e\left(\bar{u}_{\tau h}\right)-\left|e\left(\bar{u}_{\tau}\right)\right|^{\gamma-2} e\left(\bar{u}_{\tau}\right)\right): e\left(\widetilde{u}_{\tau h}-\bar{u}_{\tau}\right) \mathrm{d} x \mathrm{~d} t \\
&+\int_{\Omega} \frac{1}{2} \mathbb{D} e\left(u_{\tau h}(T)-u_{\tau}(T)\right): e\left(\widetilde{u}_{\tau h}(T)-u_{\tau}(T)\right) \mathrm{d} x \rightarrow 0 .
\end{aligned}
$$

The second inequality in (4.18) is due to the inequality $\mathbb{D} e\left(\mathrm{D}_{t} u_{\tau}^{k}\right): e\left(u_{\tau}^{k}\right) \geq \frac{1}{2} \mathrm{D}_{t}\left(\mathbb{D} e\left(u_{\tau}^{k}\right): e\left(u_{\tau}^{k}\right)\right)$, which is just a generalization of the elementary algebraic inequality of the type $\left(a_{k}-a_{k-1}\right) a_{k} \geq \frac{1}{2} a_{k}^{2}-\frac{1}{2} a_{k-1}^{2}$, together with 
the "telescopical" effect $\sum_{k=1}^{T / \tau} \frac{1}{2} a_{k}^{2}-\frac{1}{2} a_{k-1}^{2}=\frac{1}{2} a_{T / \tau}^{2}-\frac{1}{2} a_{0}^{2}$. The convergence to zero in (4.18) for $h \downarrow 0$ is because $\tau>0$ is fixed so that trivially $\mathrm{D}_{t}^{2} u_{\tau h} \rightarrow \mathrm{D}_{t}^{2} u_{\tau}$ in $L^{2}\left(Q ; \mathbb{R}^{d}\right)$ due to the Rellich compact embedding $W^{1,2}(\Omega) \Subset$ $L^{2}(\Omega)$, and furthermore also $\Theta\left(\bar{w}_{\tau h}\right) \rightarrow \Theta\left(\bar{w}_{\tau}\right)$ certainly in $L^{2}(Q)$ (in fact even in a much smaller Lebesgue space $L^{2 d \omega /(d-2)-\epsilon}(Q)$ with $\left.\epsilon>0\right)$ due to the compact embedding $W^{1,2}(\Omega)$ and $\bar{\pi}_{\tau h} \rightarrow \bar{\pi}_{\tau}$ in $L^{2}\left(Q ; \mathbb{R}^{d \times d}\right)$ due to the compact embedding $W^{a, 2}(\Omega)$ so that $\left(\bar{\pi}_{\tau h}+\mathbb{C} \Theta\left(w_{\tau h}\right) \mathbb{E}\right): e\left(\bar{u}_{\tau h}-\bar{u}_{\tau}\right) \rightarrow 0$ weakly in $L^{1}(Q)$.

Then, we use the strong monotonicity of $e \mapsto \mathbb{D} e$ to estimate, for some $c>0$,

$$
\begin{aligned}
c \| & \frac{\partial e\left(u_{\tau h}-u_{\tau}\right)}{\partial t} \|_{L^{2}\left(Q ; \mathbb{R}^{d \times d}\right)}^{2} \leq \int_{Q} \mathbb{D} \frac{\partial e\left(u_{\tau h}-u_{\tau}\right)}{\partial t}: \frac{\partial e\left(u_{\tau h}-u_{\tau}\right)}{\partial t} \mathrm{~d} x \mathrm{~d} t \\
\leq & \int_{Q} \mathbb{D} \frac{\partial e\left(u_{\tau h}-u_{\tau}\right)}{\partial t}: \frac{\partial e\left(u_{\tau h}-u_{\tau}\right)}{\partial t} \mathrm{~d} x \mathrm{~d} t+\int_{\Omega} \frac{1}{2} \mathbb{C} e\left(u_{\tau h}(T)-u_{\tau}(T)\right): e\left(u_{\tau h}(T)-u_{\tau}(T)\right) \mathrm{d} x \\
= & \int_{Q}-\varrho \mathrm{D}_{t}^{2} u_{\tau h} \frac{\partial\left(u_{\tau h}-\widetilde{u}_{\tau h}\right)}{\partial t}-\left(\mathbb{C} \pi_{\tau h}+\mathbb{C} \Theta\left(w_{\tau h}\right) \mathbb{E}+\mathbb{D} \frac{\partial e\left(u_{\tau}\right)}{\partial t}+\tau\left|e\left(u_{\tau h}\right)\right|^{\gamma-2} e\left(u_{\tau h}\right)\right): \frac{\partial e\left(u_{\tau h}-\widetilde{u}_{\tau h}\right)}{\partial t} \mathrm{~d} x \mathrm{~d} t \\
& +\int_{\Sigma} \bar{g}_{\tau} \cdot \frac{\partial\left(u_{\tau h}-\widetilde{u}_{\tau h}\right)}{\partial t} \mathrm{~d} S \mathrm{~d} t-\int_{\Omega} \frac{1}{2} \mathbb{C} e\left(u_{\tau}(T)\right): e\left(u_{\tau h}(T)-u_{\tau}(T)\right) \mathrm{d} x+\int_{Q} \mathbb{D} \frac{\partial e\left(u_{\tau h}-u_{\tau}\right)}{\partial t}: \frac{\partial e\left(\widetilde{u}_{\tau h}-u_{\tau}\right)}{\partial t} \mathrm{~d} x \mathrm{~d} t \\
& +\int_{\Omega} \frac{1}{2} \mathbb{C} e\left(u_{\tau h}(T)-u_{\tau}(T)\right):\left(e\left(\widetilde{u}_{\tau h}(T)-u_{\tau}(T)\right)\right) \rightarrow 0 .
\end{aligned}
$$

The convergence to zero for $h \downarrow 0$ again relies on $\tau>0$ fixed so that again $\mathrm{D}_{t}^{2} u_{\tau h} \rightarrow \mathrm{D}_{t}^{2} u_{\tau}$, and $\pi_{\tau h} \rightarrow \pi_{\tau}$ in $L^{2}\left(Q ; \mathbb{R}^{d \times d}\right)$ due to Rellich's compact embedding $W^{1,2}(\Omega) \Subset L^{2}(\Omega)$, and because that the strong convergence $e\left(u_{\tau h}^{k}\right) \rightarrow e\left(u_{\tau}^{k}\right)$ in $L^{\gamma}\left(Q ; \mathbb{R}^{d \times d}\right)$ has already been proved.

Furthermore, we use again the compact embedding $W^{a, 2}(\Omega) \Subset L^{2}(\Omega)$ so that, thanks to the regularizing operator $\mathcal{S}$, we have convergence also in

$$
\zeta_{1}\left(\mathrm{D}_{t} \pi_{\tau h}, \mathrm{D}_{t} \eta_{\tau h}\right) \rightarrow \zeta_{1}\left(\mathrm{D}_{t} \pi_{\tau}, \mathrm{D}_{t} \eta_{\tau}\right) \quad \text { strongly in } L^{\infty}\left(I ; L^{2}(\Omega)\right)
$$

because $\tau>0$ is considered fixed. Altogether, we proved strong convergence of the heat sources in $L^{1}(Q)$.

Then, using still the weak upper semicontinuity argument for $\mathcal{S}$-terms in (4.10b) summed over particular time levels, the claimed limit passage from (4.10) to the boundary-value problem (4.16)-(4.17) formulated weakly is easy to be seen. In particular, the limit passage from $(4.10 \mathrm{~b})$ to $(4.16 \mathrm{~b})$ uses also the approximation property $(4.9 \mathrm{~b})$.

Note that (4.2c) has the right-hand side in $L^{2}(\Omega)$ since $\gamma \geq 4$ and since $\pi_{\tau}^{k}-\pi_{\tau}^{k-1}$ and $\eta_{\tau}^{k}-\eta_{\tau}^{k-1}$ are certainly in $L^{2}\left(\Omega ; \mathbb{R}^{d \times d}\right)$ and $L^{2}(\Omega)$, respectively, hence the weak formulation of (4.2c) is understood standardly.

Let us abbreviate the regularized stored energy by $\Phi_{\tau}$, i.e.

$$
\begin{aligned}
\Phi_{\tau}(u, \pi, \eta): & =\Phi(u, \pi, \eta)+\frac{\tau}{\gamma}\|e(u)\|_{L^{\gamma}\left(\Omega ; \mathbb{R}^{d \times d)}\right.}^{\gamma}+\frac{\tau}{2}|(\pi, \eta)|_{W^{a, 2}\left(\Omega ; \mathbb{R}^{d \times d+1}\right)}^{2} \\
& =\int_{\Omega} \frac{1}{2} \mathbb{C}(e(u)-\pi):(e(u)-\pi)+\frac{1}{2} \mathbb{H} \pi: \pi+\frac{b}{2} \eta^{2}+\frac{\tau}{\gamma}|e(u)|^{\gamma} \mathrm{d} x+\frac{\tau}{2}|\pi|_{W^{a, 2}\left(\Omega ; \mathbb{R}^{d \times d}\right)}^{2}+\frac{\tau}{2}|\eta|_{W^{a, 2}(\Omega)}^{2}
\end{aligned}
$$


Lemma 4.3 (still further a-priori information). For any $k=1, \ldots, K_{\tau}$, the following "discrete mechanical energy" balance holds:

$$
\begin{aligned}
& T_{\text {kin }}\left(\mathrm{D}_{t} u_{\tau}^{k}\right)+\Phi_{\tau}\left(u_{\tau}^{k}, \pi_{\tau}^{k}, \eta_{\tau}^{k}\right)+\tau \sum_{l=1}^{k} \int_{\Omega} \zeta_{1}\left(\mathrm{D}_{t} \pi_{\tau}^{k}, \mathrm{D}_{t} \eta_{\tau}^{k}\right)+\mathbb{D} e\left(\mathrm{D}_{t} u_{\tau}^{k}\right): e\left(\mathrm{D}_{t} u_{\tau}^{k}\right) \mathrm{d} x \\
& \leq T_{\text {kin }}\left(\dot{u}_{0}\right)+\Phi_{\tau}\left(u_{0, \tau}, \pi_{0, \tau}, \eta_{0, \tau}\right)+\tau \sum_{l=1}^{k}\left(\int_{\Omega} \Theta\left(w_{\tau}^{l}\right) \mathbb{E}: \mathbb{C} e\left(\mathrm{D}_{t} u_{\tau}^{k}\right) \mathrm{d} x+\int_{\Gamma} g_{\tau}^{l} \cdot \mathrm{D}_{t} u_{\tau}^{l} \mathrm{~d} S\right)
\end{aligned}
$$

as well as the following "discrete total energy" balance holds:

$$
\begin{aligned}
T_{\text {kin }}\left(\mathrm{D}_{t} u_{\tau}^{k}\right)+\Phi_{\tau}\left(u_{\tau}^{k}, \pi_{\tau}^{k}, \eta_{\tau}^{k}\right) & +\int_{\Omega} w_{\tau}^{k} \mathrm{~d} x \leq T_{\text {kin }}\left(\dot{u}_{0}\right)+\Phi_{\tau}\left(u_{0, \tau}, \pi_{0, \tau}, \eta_{0, \tau}\right)+\int_{\Omega} w_{0} \mathrm{~d} x \\
& +\tau \sum_{l=1}^{k}\left(\int_{\Gamma} g_{\tau}^{l} \cdot \mathrm{D}_{t} u_{\tau}^{l}+f_{\text {ext }, \tau}^{l} \mathrm{~d} S-\varepsilon(\tau) \int_{\Omega}\left|w_{\tau}^{l}\right|^{\beta-2} w_{\tau}^{l} \mathrm{~d} x\right)
\end{aligned}
$$

and also the "discrete semistability"

$$
\Phi_{\tau}\left(u_{\tau}^{k}, \pi_{\tau}^{k}, \eta_{\tau}^{k}\right) \leq \Phi_{\tau}\left(u_{\tau}^{k}, \tilde{\pi}, \tilde{\eta}\right)+\int_{\Omega} \xi_{1}\left(\tilde{\pi}-\pi_{\tau}^{k}, \tilde{\eta}-\eta_{\tau}^{k}\right) \mathrm{d} x
$$

holds for any $(\tilde{\pi}, \tilde{\eta}) \in W^{a, 2}\left(\Omega ; \mathbb{R}_{\mathrm{dev}}^{d \times d} \times \mathbb{R}\right)$, where $\Phi_{\tau}$ and $T_{\mathrm{kin}}$ are from (4.21) and (2.13), respectively.

Proof. Let us use a short-hand notation $z:=(\pi, \eta)$ for this proof. Taking $\left(u_{\tau}^{k}, z_{\tau}^{k}, w_{\tau}^{k}\right)$ solving (4.2), we can test $(4.2 \mathrm{a}),(4.2 \mathrm{~b})$ respectively by $\mathrm{D}_{t} u_{\tau}^{k}$ and $\mathrm{D}_{t} z_{\tau}^{k}$. By using the convexity of $\Phi_{\tau}$ from (4.21) and by summation over time steps, we obtain (4.22).

Now, to get (4.23), we still add (4.2c) tested by 1 and summed over time steps to (4.22); here it is important that the dissipative/adiabatic terms mutually cancel in the mechanical and the thermal parts.

As for (4.24), we use that (4.2b) is the sufficient (and, of course, also necessary) optimality condition for $z_{\tau}^{k}$ to minimize the convex functional $\Phi_{\tau}\left(u_{\tau}^{k}, \cdot\right)+\int_{\Omega} \zeta_{1}\left(\cdot-z_{\tau}^{k-1}\right) \mathrm{d} x$, which gives

$$
\Phi_{\tau}\left(u_{\tau}^{k}, z_{\tau}^{k}\right)+\int_{\Omega} \tau \zeta_{1}\left(\frac{z_{\tau}^{k}-z_{\tau}^{k-1}}{\tau}\right) \mathrm{d} x \leq \Phi_{\tau}\left(u_{\tau}^{k}, \tilde{z}\right)+\int_{\Omega} \tau \zeta_{1}\left(\frac{\tilde{z}-z_{\tau}^{k-1}}{\tau}\right) \mathrm{d} x
$$

for any $\tilde{z}$ and then, by using that $\zeta_{1}$ is homogeneous degree-1 and thus satisfies the triangle inequality $\zeta_{1}\left(\tilde{z}-z_{\tau}^{k-1}\right) \leq \zeta_{1}\left(\tilde{z}-z_{\tau}^{k}\right)+\zeta_{1}\left(z_{\tau}^{k}-z_{\tau}^{k-1}\right)$, which altogether gives

$$
\Phi_{\tau}\left(u_{\tau}^{k}, z_{\tau}^{k}\right) \leq \Phi_{\tau}\left(u_{\tau}^{k}, \tilde{z}\right)+\int_{\Omega} \zeta_{1}\left(\tilde{z}-z_{\tau}^{k-1}\right)-\zeta_{1}\left(z_{\tau}^{k}-z_{\tau}^{k-1}\right) \mathrm{d} x \leq \Phi_{\tau}\left(u_{\tau}^{k}, \tilde{z}\right)+\int_{\Omega} \zeta_{1}\left(\tilde{z}-z_{\tau}^{k}\right) \mathrm{d} x
$$

arriving thus just to (4.24).

Proposition 4.4 (uniform a-priori estimates). Let, beside the assumptions from Lemma 4.1, also (2.3) hold and the exponent $\omega$ from (3.9b) satisfy

$$
\omega>\frac{2 d}{d+2}
$$


Then, for some $C$ and $C_{\tau}$, it holds

$$
\begin{aligned}
& \left\|u_{\tau}\right\|_{W^{1,2}\left(I ; W^{1,2}\left(\Omega ; \mathbb{R}^{d}\right)\right)} \leq C, \\
& \left\|\bar{\pi}_{\tau}\right\|_{L^{\infty}\left(I ; L^{2}\left(\Omega ; \mathbb{R}_{\mathrm{dev}}^{d \times d}\right)\right) \cap \operatorname{BV}\left(\bar{I} ; L^{1}\left(\Omega ; \mathbb{R}_{\mathrm{dev}}^{d \times d}\right)\right)} \leq C, \\
& \left\|\bar{\eta}_{\tau}\right\|_{L^{\infty}\left(I ; L^{2}(\Omega)\right) \cap \operatorname{BV}\left(\bar{I} ; L^{1}(\Omega)\right)} \leq C, \\
& \left\|\bar{w}_{\tau}\right\|_{L^{\infty}\left(I ; L^{1}(\Omega)\right) \cap L^{r}\left(I ; W^{1, r}(\Omega)\right)} \leq C_{r} \quad \text { with any } 1 \leq r<\frac{d+2}{d+1}, \\
& \left\|\frac{\partial w_{\tau}}{\partial t}\right\|_{L^{1}\left(I ; W^{1+d, 2}(\Omega)^{*}\right)} \leq C, \\
& \left\|\varrho \frac{\partial u_{\tau}}{\partial t}\right\|_{L^{\infty}\left(I ; L^{2}\left(\Omega ; \mathbb{R}^{d}\right)\right) \cap \operatorname{BV}\left(\bar{I} ; W^{1, \infty}\left(\Omega ; \mathbb{R}^{d}\right)^{*}\right)} \leq C, \\
& \left\|u_{\tau}\right\|_{L^{\infty}\left(I ; W^{1, \gamma}\left(\Omega ; \mathbb{R}^{d}\right)\right)} \leq C \tau^{-1 / \gamma}, \\
& \left\|\bar{\pi}_{\tau}\right\|_{L^{\infty}\left(I ; W^{a, 2}\left(\Omega ; \mathbb{R}_{\mathrm{dev}}^{d \times d}\right)\right)} \leq C \tau^{-1 / 2}, \\
& \left\|\bar{\eta}_{\tau}\right\|_{L^{\infty}\left(I ; W^{a, 2}(\Omega)\right)} \leq C \tau^{-1 / 2}, \\
& \left\|\bar{w}_{\tau}\right\|_{L^{\beta}(Q)} \leq C_{\tau} \varepsilon(\tau)^{-1 / \beta} .
\end{aligned}
$$

Note that $\frac{\partial}{\partial t} u_{\tau}$ is piece-wise constant in time with possible jumps at times $t=k \tau$, so that $\varrho \frac{\partial^{2}}{\partial t^{2}} u_{\tau}$ is a measure, which is why (4.27f) involves BV-space.

Ideas of the proof. For particular details as far as (4.27a)-(4.27i) concerns, see [31], proof of Proposition 4.2; in contrast to [31], here we have used $g$ qualified as $L^{2}\left(I ; L^{q}\left(\Gamma ; \mathbb{R}^{d}\right)\right)$ in $(3.10 \mathrm{a})$ instead of a bulk force in $L^{1}\left(I ; L^{2}\left(\Omega ; \mathbb{R}^{d}\right)\right)$ and also we have admitted $\varrho=0$, which however is just a simplified case. Let us just outline the scenario: As we already got rid of the spatial discretization, from the maximum principle one can see that $w_{\tau} \geq 0$. From (4.23) then one gets the $L^{\infty}$-parts of $(4.27 \mathrm{~b})-(4.27 \mathrm{~d})$ and $(4.27 \mathrm{f})-(4.27 \mathrm{i})$. Then one uses the $L^{1}$-theory for the evolutionary heat equation $[4,5]$ based on the test by $1-1 /\left(1+\bar{w}_{\tau}\right)^{\delta}, \delta>0$, combined with the interpolation of the adiabatic term by using several-times Gagliardo-Nirenberg inequality as in [29,31], which eventually gives (4.27a) and the rest of (4.27b)-(4.27d). Then (4.27e) and the BV-part of (4.27f) follow by the already obtained estimates.

Eventually, for $\tau>0$ fixed, we already mentioned that the right-hand side of the discrete heat equation (4.2c) is in $L^{2}(\Omega)$ so that the right-hand side $\zeta_{1}\left(\frac{\partial \pi_{\tau}}{\partial t}, \frac{\partial \eta_{\tau}}{\partial t}\right)+\mathbb{D} e\left(\frac{\partial u_{\tau}}{\partial t}\right): e\left(\frac{\partial u_{\tau}}{\partial t}\right)+\Theta\left(\bar{w}_{\tau}\right) \mathbb{E}: \mathbb{C} e\left(\frac{\partial u_{\tau}}{\partial t}\right)$ of $(4.16 \mathrm{c})$, let us denote it by $\bar{r}_{\tau}$ for a moment, is in $L^{\infty}\left(I ; L^{2}(\Omega)\right)$ so that we can still test the discrete heat equation by $w_{\tau}$ to obtain $(4.27 \mathrm{j})$.

In general, $C_{\tau}$ in (4.27j) may depend not only on $\tau$ but implicitly also on $\varepsilon=\varepsilon(\tau)$ and then (4.27j) says nothing more than $w_{\tau} \in L^{\beta}(Q)$ only, unless one has some more specific information about this implicit dependence. Here, however, for $\gamma$ sufficiently large, one can show that $C_{\tau}$ even does not depend on $\varepsilon(\tau)$ at all. Let us present the calculations only for the physically relevant case, i.e. $d=3$ (while for $d \leq 2$ it is even less restrictive).

Lemma 4.5. Let $d=3$, let the assumptions from Lemma 4.1 and (4.26) hold, and let, in addition, $\gamma>76 / 17$. Then (4.27j) holds with $C_{\tau}=C \tau^{-2(\gamma+1) /(2 \beta \gamma)}$ for some $C$ independent of $\tau$.

Note that the condition $\gamma>76 / 17 \doteq 4.47$ may (but need not, depending on $\omega$ ) slightly strengthen (4.13).

Proof of Lemma 4.5. In fact, by testing the heat equation (4.2c) by $w_{\tau}^{k}$ as already used in the proof of Proposition 4.4, we can see that the constant $C_{\tau}$ in $(4.27 \mathrm{j})$ is proportional to $\left\|\bar{r}_{\tau}\right\|_{L^{2}\left(I ; L^{6 / 5}(\Omega)\right)}^{2 / \beta}$ where $\bar{r}_{\tau}$ is 
the right-hand side of (4.16c) as in the proof of Proposition 4.4. More in detail, this test gives

$$
\begin{aligned}
& \int_{Q} \kappa_{0}\left|\nabla \bar{w}_{\tau}\right|^{2}+\varepsilon(\tau)\left|\bar{w}_{\tau}\right|^{\beta} \mathrm{d} x \mathrm{~d} t \leq \int_{Q} \bar{r}_{\tau} \bar{w}_{\tau} \mathrm{d} x \mathrm{~d} t+\int_{\Sigma} \bar{w}_{\tau} \bar{f}_{\tau} \mathrm{d} S \mathrm{~d} t+\int_{\Omega} \frac{1}{2} w_{0, \tau}^{2} \mathrm{~d} x \\
& \quad \leq\left\|\bar{r}_{\tau}\right\|_{L^{2}\left(I ; L^{6 / 5}(\Omega)\right)}\left\|\bar{w}_{\tau}\right\|_{L^{2}\left(I ; L^{6}(\Omega)\right)}+\left\|\bar{f}_{\tau}\right\|_{L^{2}\left(I ; L^{4 / 3}(\Gamma)\right)}\left\|\bar{w}_{\tau}\right\|_{L^{2}\left(I ; L^{4}(\Gamma)\right)}+\left\|w_{0, \tau}\right\|_{L^{2}(\Omega)}^{2},
\end{aligned}
$$

where $\kappa_{0}>0$ denotes the infimum from (3.9c). Taking into account the already obtained $L^{\infty}\left(I ; L^{1}(\Omega)\right.$ )-estimate (4.27d), we see that the $\kappa_{0}$-term controls the full norm in $L^{2}\left(I ; W^{1,2}(\Omega)\right) \subset L^{2}\left(I ; L^{6}(\Omega)\right)$. Thus it is desirable to estimate the particular terms of $\bar{r}_{\tau}$ in $L^{2}\left(I ; L^{6 / 5}(\Omega)\right)$ independently of $\varepsilon=\varepsilon(\tau)$.

By (4.27a), we have $\left\|e\left(\frac{\partial u_{\tau}}{\partial t}\right)\right\|_{L^{2}\left(Q ; \mathbb{R}^{d \times d}\right)} \leq C$. By $(4.27 \mathrm{~g})$, we have $\left\|e\left(u_{\tau}\right)\right\|_{L^{\infty}\left(I ; L^{\gamma}\left(\Omega ; \mathbb{R}^{d \times d}\right)\right)} \leq C / \tau^{1 / \gamma}$, so that, realizing the equidistance of the partition with the time-step $\tau$, we have also $\left\|e\left(\frac{\partial u_{\tau}}{\partial t}\right)\right\|_{L^{\infty}\left(I ; L^{\gamma}\left(\Omega ; \mathbb{R}^{d \times d)}\right)\right.} \leq$ $C / \tau^{1+1 / \gamma}$. Interpolating these estimates with the weights $\frac{1}{2}$ and $\frac{1}{2}$ yields

$$
\left\|e\left(\frac{\partial u_{\tau}}{\partial t}\right)\right\|_{L^{4}\left(I ; L^{4 \gamma /(\gamma+2)}\left(\Omega ; \mathbb{R}^{d \times d}\right)\right)} \leq \frac{C}{\tau^{(\gamma+1) /(2 \gamma)}} .
$$

Thus we have the estimate for the viscous part of the dissipative heat

$$
\left\|\mathbb{C} e\left(\frac{\partial u_{\tau}}{\partial t}\right): e\left(\frac{\partial u_{\tau}}{\partial t}\right)\right\|_{L^{2}\left(I ; L^{2 \gamma /(\gamma+2)}(\Omega)\right)} \leq \frac{C}{\tau^{(\gamma+1) / \gamma}} .
$$

Note that the desired embedding $L^{2}\left(I ; L^{2 \gamma /(\gamma+2)}(\Omega)\right) \subset L^{2}\left(I ; L^{6 / 5}(\Omega)\right)$ needs here $\gamma \geq 3$, which always holds due to (4.13). As to the adiabatic heat, we use the interpolation between two estimates in (4.27d), i.e. between $L^{\infty}\left(I ; L^{1}(\Omega)\right)$ and $L^{r}\left(I ; W^{1, r}(\Omega)\right)$ with $r<5 / 4$, with the weight $\lambda$ and $1-\lambda$ with sufficiently small $\lambda>5 / 8$ to obtain $w_{\tau} \in L^{10 / 3}\left(I ; L^{q}(\Omega)\right)$ with $q<5 / 4$. As $\Theta$ has the sublinear polynomial growth with an exponent less than $5 / 6$, cf. (4.15) with $\omega>6 / 5$ due to (4.26), we then have the temperature $\Theta\left(\bar{w}_{\tau}\right) \in L^{4}\left(I ; L^{6 q / 5}(\Omega)\right)$. By (4.29), we have

$$
\left\|\Theta\left(\bar{w}_{\tau}\right) \mathbb{E}: \mathbb{C} e\left(\frac{\partial u_{\tau}}{\partial t}\right)\right\|_{L^{2}\left(I ; L^{12 \gamma q /(2 \gamma \omega+19)}(\Omega)\right)} \leq \frac{C}{\tau^{(\gamma+1) /(2 \gamma)}}
$$

Here the desired embedding $L^{4}\left(I ; L^{12 \gamma q /(2 \gamma \omega+19)}(\Omega)\right) \subset L^{2}\left(I ; L^{6 / 5}(\Omega)\right)$ is possible provided $\gamma$ is sufficiently large, namely $\gamma>76 / 17$.

Eventually, by the $L^{\infty}$-parts of $(4.27 \mathrm{~b}),(4.27 \mathrm{c})$, we have also $\left\|\frac{\partial \pi_{\tau}}{\partial t}\right\|_{L^{\infty}\left(I ; L^{2}\left(\Omega ; \mathbb{R}^{d \times d+1}\right)\right)} \leq C / \tau$ which can be still interpolated (with the weight $\frac{1}{2}$ and $\left.\frac{1}{2}\right)$ with $\left\|\frac{\partial \pi_{\tau}}{\partial t}\right\|_{L^{1}\left(I ; L^{1}\left(\Omega ; \mathbb{R}^{d \times d+1}\right)\right)} \leq C$ due to the BV-part of (4.27b), and similarly for $\frac{\partial \pi_{\tau}}{\partial t}$ due to the BV-part of $(4.27 \mathrm{c})$, so that the remaining contribution to the dissipative heat can be estimated as

$$
\left\|\zeta_{1}\left(\frac{\partial \pi_{\tau}}{\partial t}, \frac{\partial \eta_{\tau}}{\partial t}\right)\right\|_{L^{2}\left(I ; L^{4 / 3}(\Omega)\right)} \leq \frac{C}{\tau^{1 / 2}} .
$$

The last two terms in (4.28) are of the same order $\mathcal{O}\left(\tau^{-1 / 2}\right)$ as (4.32) due to (4.8d) and (4.8e). Thus

$$
C_{\tau} \sim\left\|\bar{r}_{\tau}\right\|_{L^{2}\left(I ; L^{6 / 5}(\Omega)\right)}^{2 / \beta}=\mathcal{O}\left(\frac{1}{\tau^{2(\gamma+1) /(2 \beta \gamma)}}\right) .
$$


Proposition 4.6 (convergence for $\tau \downarrow 0)$. Let $\varepsilon=\varepsilon(\tau)$ be chosen to converge to 0 for $\tau \downarrow 0$ sufficiently fast so that

$$
\lim _{\tau \downarrow 0} C_{\tau}^{\beta-1} \varepsilon(\tau)^{1 / \beta}=0,
$$

with $C_{\tau}$ referring to $(4.27 \mathrm{j})$. Then there is a subsequence of $\left\{\left(u_{\tau}, \pi_{\tau}, \eta_{\tau}, w_{\tau}\right)\right\}_{\tau>0}$ weakly* convergent in the topologies indicated in (4.27a)-(4.27f), cf. Remark 4.7, to some $(u, \pi, \eta, w)$ and, if the initial conditions $\left(\pi_{0}, \eta_{0}\right)$ are semistable with respect to $u_{0}$ in the sense

$$
\Phi\left(u_{0}, \pi_{0}, \eta_{0}\right) \leq \Phi\left(u_{0}, \tilde{\pi}, \tilde{\eta}\right)+\int_{\Omega} \zeta_{1}\left(\tilde{\pi}-\pi_{0}, \tilde{\eta}-\eta_{0}\right) \mathrm{d} x
$$

for all $(\tilde{\pi}, \tilde{\eta}) \in L^{2}\left(\Omega ; \mathbb{R}_{\mathrm{dev}}^{d \times d} \times \mathbb{R}\right)$, then $(u, \pi, \eta, w)$ is an energetic solution according Definition 3.1 .

Sketch of the proof. For some details see [31], proof of Proposition 4.3 and Remark 4.5, the essential differences are the regularizing $\mathcal{S}$ - and $\beta$-terms. Let us again use the short-hand notation $z:=(\pi, \eta)$ for this proof.

First, by Banach's selection principle, we select a weakly* convergent subsequence. By the generalized Helly principle $\bar{z}_{\tau}(t) \rightarrow z(t)=(\pi(t), \eta(t))$ weakly in $L^{2}\left(\Omega ; \mathbb{R}_{\mathrm{dev}}^{d \times d} \times \mathbb{R}\right)$ for all $t \in[0, T]$ as well as $\bar{u}_{\tau}(t) \rightarrow u(t)$ weakly in $W^{1,2}\left(\Omega ; \mathbb{R}^{d}\right)$, and also $\bar{w}_{\tau}(t) \rightarrow w(t)$ weakly* in $\mathcal{M}(\bar{\Omega})$.

To pass to the limit in (4.16a) by-part integrated over $I$ to the weakly formulated momentum equation (3.7a) is simple because all terms are either linear, or enjoy compactness (which concerns $\Theta(w)$-term), or vanish due to the estimate $(4.27 \mathrm{~g})$ since

$$
\left.\left|\tau \int_{Q}\right| e\left(\bar{u}_{\tau}\right)\right|^{\gamma-2} e\left(\bar{u}_{\tau}\right): e(v) \mathrm{d} x \mathrm{~d} t \mid \leq \tau\left\|e\left(\bar{u}_{\tau}\right)\right\|_{L^{\gamma}\left(Q ; \mathbb{R}^{d \times d}\right)}^{\gamma-1}\|e(v)\|_{L^{\gamma}\left(Q ; \mathbb{R}^{d \times d}\right)}=\mathcal{O}\left(\tau^{1 / \gamma}\right) \rightarrow 0
$$

To pass to the limit in the semi-stability (4.24) towards (3.7e), we need to construct a so-called joint-recovery sequence, $c f$. [25]. Here it essentially means that, for any $\hat{z}=(\hat{\pi}, \hat{\eta}) \in L^{2}\left(\mathbb{R}_{\text {dev }}^{d \times d} \times \mathbb{R}\right)$ with $\hat{\eta}-\eta(t) \geq \delta_{P_{0}}^{*}(\hat{\pi}-\pi(t))$, we need to find a sequence $\hat{z}_{\tau}=\left(\hat{\pi}_{\tau}, \hat{\eta}_{\tau}\right)$ in $W^{a, 2}\left(\Omega ; \mathbb{R}^{d \times d+1}\right)$ such that

$$
\limsup _{\tau \downarrow 0} \Phi_{\tau}\left(u_{\tau}(t), \hat{z}_{\tau}\right)-\Phi_{\tau}\left(u_{\tau}(t), z_{\tau}(t)\right)+\int_{\Omega} \xi_{1}\left(\hat{z}_{\tau}-z_{\tau}(t)\right) \mathrm{d} x \leq \Phi(u(t), \hat{z})-\Phi(u(t), z(t))+\int_{\Omega} \xi_{1}(\hat{z}-z(t)) \mathrm{d} x .
$$

In fact, the true Gibbs' stored energy would still yield the term $\int_{\Gamma} g_{\tau}(t) \cdot\left(\hat{z}_{\tau}-z_{\tau}(t)\right) \mathrm{d} S$ which, however, could easily be shown to converge to zero if the construction (4.37) below is adopted.

Let us denote the standard mollifier $[\cdot]_{\delta}$ by convolution with a standard positive kernel whose support is of diameter proportional to $\delta$. Thus we can rely on $\left\|[z]_{\delta}\right\|_{W^{1,2}(\Omega)} \leq C \delta^{-1}\|z\|_{L^{2}(\Omega)}$ so that, by interpolation with $\left\|[z]_{\delta}\right\|_{L^{2}(\Omega)} \leq C\|z\|_{L^{2}(\Omega)}$, one gets $\left\|[z]_{\delta}\right\|_{W^{a, 2}(\Omega)} \leq C \delta^{-a}\|z\|_{L^{2}(\Omega)}$. Then, for $z \in L^{2}(\Omega)$, we have also $[z]_{\delta} \rightarrow z$ in $L^{2}(\Omega)$ for $\delta \rightarrow 0$. We take the joint-recovery sequence as

$$
\hat{z}_{\tau}:=z_{\tau}(t)+[\hat{z}-z(t)]_{\delta(\tau)} \quad \text { with } \delta(\tau):=\tau^{1 /(4 a)} .
$$

We rely on the quadratic form of $\Phi_{\tau}$, which by the binomial formula results to

$\Phi_{\tau}(u, \hat{z})-\Phi_{\tau}(u, z)=\int_{\Omega} \frac{1}{2}(\mathbb{C}+\mathbb{H})(\hat{\pi}-\pi):(\hat{\pi}+\pi)-\mathbb{C} e(u):(\hat{\pi}-\pi)+\frac{b}{2}(\hat{\eta}-\eta)(\hat{\eta}+\eta)+\frac{\tau}{2} \mathcal{S}^{1 / 2}(\hat{z}-z): \mathcal{S}^{1 / 2}(\hat{z}+z) \mathrm{d} x$.

By (4.37), we have

$$
\hat{z}_{\tau}-z_{\tau}(t)=[\hat{z}-z(t)]_{\delta(\tau)} \rightarrow \hat{z}-z(t) \quad \text { strongly in } L^{2}\left(\Omega ; \mathbb{R}^{d \times d+1}\right),
$$


which causes the convergence

$$
\begin{aligned}
(\mathbb{C}+\mathbb{H}) \hat{\pi}_{\tau}: \hat{\pi}_{\tau}-(\mathbb{C}+\mathbb{H}) \pi_{\tau}(t): \pi_{\tau}(t) & =(\mathbb{C}+\mathbb{H})\left(\hat{\pi}_{\tau}-\pi_{\tau}(t)\right):\left(\hat{\pi}_{\tau}+\pi_{\tau}(t)\right)=(\mathbb{C}+\mathbb{H})[\hat{\pi}-\pi(t)]_{\delta(\tau)}:\left(\hat{\pi}_{\tau}+\pi_{\tau}(t)\right) \\
& \rightarrow(\mathbb{C}+\mathbb{H})(\hat{\pi}-\pi(t)):(\hat{\pi}+\pi(t))=(\mathbb{C}+\mathbb{H}) \hat{\pi}: \hat{\pi}-(\mathbb{C}+\mathbb{H}) \pi(t): \pi(t)
\end{aligned}
$$

weakly in $L^{1}(\Omega)$. Similarly, we can converge the term $\frac{b}{2}\left|\hat{\eta}_{\tau}\right|^{2}-\frac{b}{2}\left|\eta_{\tau}(t)\right|^{2}=\frac{b}{2}\left(\hat{\eta}_{\tau}-\eta_{\tau}(t)\right)\left(\hat{\eta}_{\tau}+\eta_{\tau}(t)\right)$. The further term in the difference $\Phi_{\tau}\left(u_{\tau}(t), \hat{z}_{\tau}\right)-\Phi_{\tau}\left(u_{\tau}(t), z_{\tau}(t)\right)$ in (4.36) admits the limit

$$
\mathbb{C} e\left(u_{\tau}(t)\right):\left(\hat{\pi}_{\tau}-\pi_{\tau}(t)\right)=\mathbb{C} e\left(u_{\tau}(t)\right):[\hat{\pi}-\pi(t)]_{\delta(\tau)} \rightarrow \mathbb{C} e(u(t)):(\hat{\pi}-\pi(t))
$$

weakly in $L^{1}(\Omega)$, where we used (4.39). Moreover, to limit the $\mathcal{S}$-term in (4.36), by (4.6) we have $|z|_{W^{a, 2}(\Omega)}=$ $\left\|\mathcal{S}^{1 / 2} z\right\|_{L^{2}(\Omega)}$ and, by the choice of $\delta(\tau)$ in $(4.37)$, we have also $\left\|[z]_{\delta(\tau)}\right\|_{W^{a, 2}(\Omega)}=\mathcal{O}\left(\tau^{-1 / 4}\right)$. This implies that

$$
\begin{aligned}
\left\|\mathcal{S}^{1 / 2}\left(\hat{z}_{\tau}-z_{\tau}(t)\right)\right\|_{L^{2}\left(\Omega ; \mathbb{R}^{d \times d+1}\right)} & =\left\|\mathcal{S}^{1 / 2}\left([\hat{z}-z(t)]_{\delta(\tau)}\right)\right\|_{L^{2}\left(\Omega ; \mathbb{R}^{d \times d+1}\right)} \\
& =\left\|\mathcal{S}^{1 / 2}\right\|_{\mathcal{L}\left(W^{a, 2}(\Omega), L^{2}(\Omega)\right)}\left\|[\hat{z}-z(t)]_{\delta(\tau)}\right\|_{W^{a, 2}\left(\Omega ; \mathbb{R}^{d \times d+1}\right)}=\mathcal{O}\left(\tau^{-1 / 4}\right)
\end{aligned}
$$

while

$$
\left\|\mathcal{S}^{1 / 2}\left(\hat{z}_{\tau}+z_{\tau}(t)\right)\right\|_{L^{2}\left(\Omega ; \mathbb{R}^{d \times d+1}\right)}=\left\|\mathcal{S}^{1 / 2}\left(2 z_{\tau}(t)+[\hat{z}-z(t)]_{\delta(\tau)}\right)\right\|_{L^{2}\left(\Omega ; \mathbb{R}^{d \times d+1}\right)}=\mathcal{O}\left(\tau^{-1 / 2}\right)
$$

due to $(4.27 \mathrm{~h}),(4.27 \mathrm{i})$. Thus the remaining term in the difference $\Phi_{\tau}\left(u_{\tau}(t), \hat{z}_{\tau}\right)-\Phi_{\tau}\left(u_{\tau}(t), z_{\tau}(t)\right)$ in $(4.36)$ can be estimated as

$$
\int_{\Omega} \tau \mathcal{S}^{1 / 2}\left(\hat{z}_{\tau}-z_{\tau}(t)\right): \mathcal{S}^{1 / 2}\left(\hat{z}_{\tau}+z_{\tau}(t)\right) \mathrm{d} x=\mathcal{O}\left(\tau^{1 / 4}\right) \rightarrow 0
$$

Still we need to pass in the $\xi_{1}$-term in (4.36) but, by (4.39), we have also $\xi_{1}\left(\hat{z}_{\tau}-z_{\tau}(t)\right)=\xi_{1}\left([\hat{z}-z(t)]_{\delta(\tau)}\right) \rightarrow$ $\xi_{1}(z-z(t))$ certainly in $L^{1}(\Omega)$ (in fact even in $L^{2}(\Omega)$ ). Here we also used that, as the kernel in the mollifier is positive, $[\cdot]_{\delta}$ remains in the convex set $\operatorname{dom}\left(\xi_{1}\right)$, hence $\xi_{1}\left(\hat{z}_{\tau}-z_{\tau}(t)\right)<\infty$ a.e. on $\Omega$ provided $\xi_{1}(\hat{z}-z(t))<\infty$. Altogether, we can show (4.36) even as an equality with "lim", and thus we also proved the semistability (3.7e) instead of just mere inequality with "limsup".

The limit passage in the energy inequality (4.23) for $k=K_{\tau}$ to $(3.7 \mathrm{~d})$ with " $\leq$ " is due to weak lower semicontinuity together with the convergence $\Phi_{\tau}\left(u_{0, \tau}, z_{0, \tau}\right) \rightarrow \Phi\left(u_{0}, z_{0}\right)$ which uses (4.8a)-(4.8c).

Having already proved the semistability (3.7e), we can show the lower energy estimate (3.7d) with " $\geq$ " by a Riemann-sum approximation of Lebesgue integral and thus energy equality as far as $z$-component concerns, i.e.

$$
\Phi(u(T), z(T))+\operatorname{Var}_{\zeta_{1}}(z ; 0, T) \geq \Phi\left(u_{0}, z_{0}\right)+\int_{Q} \mathbb{C}(e(u)-\pi): e\left(\frac{\partial u}{\partial t}\right) \mathrm{d} x \mathrm{~d} t
$$

for this rather technical argument we refer to [12], cf. also [21], or in this "semi-stable" context rather to [31], Step 7 in the proof of Proposition 4.3. Here it is also important that we have already proved (3.7a), from which we can also get the information $\frac{\partial^{2}}{\partial t^{2}} u \in L^{2}\left(I ; W^{1,2}\left(\Omega ; \mathbb{R}^{d}\right)^{*}\right)$ (which does not follow directly from (4.27f)), and then we can test it by $v:=\frac{\partial}{\partial t} u$ which is in duality with $\frac{\partial^{2}}{\partial t^{2}} u$ to get the energy balance (as an equality) as far as the $u$-component concerns. By summing it with (4.45), we thus obtain the mechanical energy balance 
(cf. (4.22)) with " $\geq "$, i.e.

$$
\begin{aligned}
T_{\text {kin }}\left(\frac{\partial u}{\partial t}(T)\right)+\Phi(u(T), z(T))+\operatorname{Var}_{\zeta_{1}}(z ; 0, T)+\int_{\bar{Q}} \mathbb{D} e\left(\frac{\partial u}{\partial t}\right): e\left(\frac{\partial u}{\partial t}\right) \mathrm{d} x & \\
\geq & T_{\text {kin }}\left(\dot{u}_{0}\right)+\Phi\left(u_{0}, z_{0}\right)+\int_{Q} \Theta(w) \mathbb{E}: \mathbb{C} e\left(\frac{\partial u}{\partial t}\right) \mathrm{d} x+\int_{\Gamma} g \cdot \frac{\partial u}{\partial t} \mathrm{~d} S
\end{aligned}
$$

Now, referring to the measure $\mathfrak{h}_{z}$ corresponding to $\zeta_{1}\left(\frac{\partial z}{\partial t}\right)$ defined in $(3.7 \mathrm{c})$, then like in [31] we have

$$
\begin{aligned}
& \int_{\bar{Q}} \mathfrak{h}_{z}(\mathrm{~d} x \mathrm{~d} t)+2 \int_{Q} \zeta_{2}\left(e\left(\frac{\partial u}{\partial t}\right)\right) \mathrm{d} x \mathrm{~d} t=\operatorname{Var}_{\zeta_{1}}(z ; 0, T)+2 \int_{Q} \zeta_{2}\left(e\left(\frac{\partial u}{\partial t}\right)\right) \mathrm{d} x \mathrm{~d} t \\
& \leq \liminf _{\tau \downarrow 0} \int_{Q} \zeta_{1}\left(\frac{\partial z_{\tau}}{\partial t}\right)+2 \zeta_{2}\left(e\left(\frac{\partial u_{\tau}}{\partial t}\right)\right) \mathrm{d} x \mathrm{~d} t \leq \limsup _{\tau \downarrow 0} \int_{Q} \zeta_{1}\left(\frac{\partial z_{\tau}}{\partial t}\right)+2 \zeta_{2}\left(e\left(\frac{\partial u_{\tau}}{\partial t}\right)\right) \mathrm{d} x \mathrm{~d} t \\
& \quad \leq \limsup _{\tau \downarrow 0}\left(\int_{\Omega} \frac{\varrho}{2}\left|\dot{u}_{0}\right|^{2}-\frac{\varrho}{2}\left|\frac{\partial u_{\tau}}{\partial t}(T)\right|^{2} \mathrm{~d} x+\Phi_{\tau}\left(u_{0, \tau}, z_{0, \tau}\right)\right. \\
& \left.\quad-\Phi_{\tau}\left(u_{\tau}(T), z_{\tau}(T)\right)+\int_{Q} \Theta\left(\bar{w}_{\tau}\right) \mathbb{E}: \mathbb{C} e\left(\frac{\partial u_{\tau}}{\partial t}\right) \mathrm{d} x \mathrm{~d} t-\int_{\Sigma} \bar{g}_{\tau} \cdot \frac{\partial u_{\tau}}{\partial t} \mathrm{~d} S \mathrm{~d} t\right) \\
& \leq \int_{\Omega} \frac{\varrho}{2}\left|\dot{u}_{0}\right|^{2}-\frac{\varrho}{2}\left|\frac{\partial u}{\partial t}(T)\right|^{2} \mathrm{~d} x+\Phi\left(u_{0}, z_{0}\right)-\Phi(u(T), z(T))+\int_{Q} \Theta(w) \mathbb{E}: \mathbb{C} e\left(\frac{\partial u}{\partial t}\right) \mathrm{d} x \mathrm{~d} t-\int_{\Sigma} g \cdot \frac{\partial u}{\partial t} \mathrm{~d} S \mathrm{~d} t \\
& \leq \operatorname{Var}_{\zeta_{1}}(z ; 0, T)+2 \int_{Q} \zeta_{2}\left(e\left(\frac{\partial u}{\partial t}\right)\right) \mathrm{d} x \mathrm{~d} t .
\end{aligned}
$$

The inequalities in (4.47) are successively by the lower weak* semicontinuity, by general comparison "liminf $\leq$ limsup", by the discrete mechanical-energy inequality (4.22) for $k=K_{\tau}$, by the upper weak* semicontinuity and the obvious non-negativity $\Phi_{\tau}-\Phi \geq 0$ and by the convergence

$$
\Theta\left(\bar{w}_{\tau}\right) \mathbb{E}: \mathbb{C} e\left(\frac{\partial u_{\tau}}{\partial t}\right) \rightarrow \Theta(w) \mathbb{E}: \mathbb{C} e\left(\frac{\partial u}{\partial t}\right) \quad \text { weakly in } L^{1}(Q)
$$

and also by (4.8b) so that $\tau\left|z_{0, \tau}\right|_{W^{a, 2}\left(\Omega ; \mathbb{R}^{d \times d+1}\right)}^{2} \rightarrow 0$, and finally by (4.46). Thus we have equality in the above chain of inequalities (4.47). This allows us to say that $\zeta_{1}\left(\frac{\partial z_{\tau}}{\partial t}\right) \rightarrow \mathfrak{h}_{z}$ weakly* in measures on $\bar{Q}$ and $\zeta_{2}\left(\frac{\partial e\left(u_{\tau}\right)}{\partial t}\right) \rightarrow \zeta_{2}\left(\frac{\partial e(u)}{\partial t}\right)$ even strongly in $L^{1}(Q)$.

This allows for the limit passage in the enthalpy equation. In addition, by using (4.34), we also get rid of the regularizing $\beta$-term. More specifically, for any smooth $z$, we can estimate this term by using (4.27j) as

$$
\left.\left|\int_{Q} \varepsilon(\tau)\right| \bar{w}_{\tau}\right|^{\beta-2} \bar{w}_{\tau} z \mathrm{~d} x \mathrm{~d} t \mid \leq \varepsilon(\tau)\left\|\bar{w}_{\tau}\right\|_{L^{\beta}(Q)}^{\beta-1}\|z\|_{L^{\beta}(Q)} \leq \varepsilon(\tau)\left(C_{\tau} \varepsilon(\tau)^{-1 / \beta}\right)^{\beta-1}=C_{\tau}^{\beta-1} \varepsilon(\tau)^{1 / \beta} \rightarrow 0 .
$$

Having (3.7b) already at disposal, we also obtain (3.6) and we can test (3.7b) by $v:=1$ which is obviously in duality with $\frac{\partial w}{\partial t} \in L^{1}\left(I ; W^{1+d}(\Omega)^{*}\right)$, and summing it with (4.46), we obtain (3.7d) with " $\geq$ ". As the opposite inequality has already been discussed, altogether we proved the total energy equality $(3.7 \mathrm{~d})$.

Remark 4.7. The weak* topologies mentioned in Proposition 4.6 are meant, of course, in suitably extended spaces because $(4.27 \mathrm{~b}),(4.27 \mathrm{c}),(4.27 \mathrm{e})$ involves $L^{1}$-spaces on which weak* topology is not defined at all. 
As to $(4.27 \mathrm{e})$, we consider $\mathcal{M}\left(\bar{I} ; W^{1+d, 2}(\Omega)^{*}\right)$ rather than $L^{1}\left(I ; W^{1+d, 2}(\Omega)^{*}\right)$, as used already in (3.6f). As to $(4.27 \mathrm{~b}),(4.27 \mathrm{c})$, we enlarge $\mathcal{M}\left(\bar{I} ; L^{1}\left(\Omega ; \mathbb{R}_{\mathrm{dev}}^{d \times d}\right)\right)$ and $\mathcal{M}\left(\bar{I} ; L^{1}(\Omega)\right)$ to the Borel measures $\mathcal{M}\left(\bar{I} \times \bar{\Omega} ; \mathbb{R}_{\mathrm{dev}}^{d \times d}\right)$ and $\mathcal{M}(\bar{I} \times \bar{\Omega})$ so that the rate of plastic deformation $\frac{\partial \pi_{\tau}}{\partial t}$ and hardening $\frac{\partial \eta_{\tau}}{\partial t}$ are a-priori bounded in $C\left(\bar{I} \times \bar{\Omega} ; \mathbb{R}_{\mathrm{dev}}^{d \times d}\right)^{*}$ and $C(\bar{I} \times \bar{\Omega})^{*}$, respectively. Then, after having the information that the limit is a solution, one can a-posteriori obtain the $L^{1}$-information as far as $\pi(t)$ and $\eta(t)$ concern.

Corollary 4.8 (conditional convergence for $h \downarrow 0$ and $\tau \downarrow 0$ ). Let $d \leq 3$, let the assumptions from Lemma 4.1 and (4.26) hold with $\gamma>76 / 17$, let (4.35) hold, and let

$$
\varepsilon(\tau)=o\left(\tau^{(\gamma+1)(\beta-1) /\left(\beta^{2} \gamma\right)}\right)
$$

Then:

(i) The convergence (in terms of subsequences) of the weak solutions to (4.16)-(4.17) with (4.4) towards energetic solutions according Definition 3.1 for $\tau \downarrow 0$, claimed in Proposition 4.6, holds.

(ii) There is a function $H: \mathbb{R}^{+} \rightarrow \mathbb{R}^{+}$such that every subsequence in the set $\left\{\left(u_{\tau h}, \pi_{\tau h}, \eta_{\tau h}, w_{\tau h}\right)\right\}_{h>0, \tau>0, h \leq H(\tau)}$ of the Galerkin approximate solutions obtained by (4.10) which converges for $h \downarrow 0$ and $\tau \downarrow 0$ weakly* in the topologies indicated in (4.27a)-(4.27f) yields, as its limit $(u, \pi, \eta, w)$, an energetic solution according Definition 3.1.

Proof. Note that, using Lemma 4.5, we have (4.33) which, together with (4.50), guarantees (4.34). Then Proposition 4.6 guarantees the claimed convergence.

To prove (ii), let us first note that all spaces involved in (4.27a)-(4.27f) have separable preduals; here we again have in mind the extension of the $L^{1}$-space occurring in $(4.27 \mathrm{~b}),(4.27 \mathrm{c}),(4.27 \mathrm{e})$ as in Remark 4.7 . In this way, we ensure all occurring weak* topologies to be compact and metrizable if restricted on any closed ball $B_{\rho}(0)$ centered at the origin 0 of the radius $\rho$ referring to norms in $(4.27 \mathrm{a})-(4.27 \mathrm{f})$. We use $\rho$ so large that all estimates (4.27a)-(4.27f) yield a subset of $B_{\rho-1}(0)$; as to $(4.27 \mathrm{~d})$, we can consider just one $r$ which is sufficiently large (with respect to $\omega$ ) that is used for interpolation which yields $(4.27 \mathrm{~d})$, cf. again $[29,31]$ for details.

Then we consider the set $\mathfrak{S}_{0}$ of all energetic solution in accord to Definition 3.1 which lie $B_{\rho-1}(0)$. We have already proved that $\mathfrak{S}_{0}$ is non-empty. Similarly, for $\tau>0$, we consider the set $\mathfrak{S}_{\tau}$ of the solutions $\left(u_{\tau}, \pi_{\tau}, \eta_{\tau}, w_{\tau}\right) \in B_{\rho-1}(0)$ to the problem (4.16)-(4.17) with (4.4). In Lemmas 4.1-4.2 and Proposition 4.4, we proved that the sets $\mathfrak{S}_{\tau}$ are nonempty for any $\tau>0$. Then, considering again the metric generating the mentioned weak* topology on $B_{\rho}(0)$, we denote by $N_{\epsilon}(S) \subset B_{\rho}(0)$ a $\epsilon$-neighbourhood of a set $S \subset B_{\rho}(0)$, i.e. $N_{\epsilon}(S):=\cup_{s \in S} \mathscr{N}_{\epsilon}(s) \cap B_{\rho}(0)$ where $\mathscr{N}_{\epsilon}(s)$ is an $\epsilon$-neighbourhood of $s$ with respect to the above mentioned metric. Note that $N_{\epsilon}(S)$, being a union of open sets, is always open in $B_{\rho}(0)$ and thus $B_{\rho}(0) \backslash N_{\epsilon}(S)$ is always compact, if nonempty.

For all $\epsilon>0$, there is $\tau_{\epsilon}>0$ such that $\mathfrak{S}_{\tau} \subset N_{\epsilon}\left(\mathfrak{S}_{0}\right)$ for all $0<\tau \leq \tau_{\epsilon}$; indeed, if $\epsilon$ is so large that $N_{\epsilon}\left(\mathfrak{S}_{0}\right)=B_{\rho}(0)$, there is nothing to prove since always $\mathfrak{S}_{\tau} \subset B_{\rho}(0)$, while in the opposite case, supposing the contrary, we would find a sequence in the nonempty compact set $B_{\rho}(0) \backslash N_{\epsilon}\left(\mathfrak{S}_{0}\right)$ and, again by arguments as in Proposition 4.6, we could show that (even all) its cluster point(s) for $\tau \rightarrow 0$ would again be the solution(s), i.e. belong to $\mathfrak{S}_{0}$, which is however a contradiction with being in $B_{\rho}(0) \backslash N_{\epsilon}\left(\mathfrak{S}_{0}\right) \subset B_{\rho}(0) \backslash \mathfrak{S}_{0}$. Beside, we can assume $\tau_{\epsilon} \rightarrow 0$ for $\epsilon \rightarrow 0$, e.g. $\tau_{\epsilon} \leq \epsilon$.

Let us now denote by $\mathfrak{S}_{\tau h}$ the set of the solutions $\left(u_{\tau h}, \pi_{\tau h}, \eta_{\tau h}, w_{\tau h}\right)$ whose existence has been proved in Lemma 4.1. It should be emphasized that we even cannot exclude that $\mathfrak{S}_{\tau h} \cap B_{\rho}(0)=\emptyset$. Anyhow, fixing $\tau>0$, we can show that there is $H(\tau)>0$ such that, for any $0<h \leq H(\tau)$, even $\mathfrak{S}_{\tau h} \subset N_{\tau}\left(\mathfrak{S}_{\tau}\right)$. Assume the contrary, i.e. for each $H>0$ one can find some $0<h_{H} \leq H$ such that $\left(u_{\tau h_{H}}, \pi_{\tau h_{H}}, \eta_{\tau h_{H}}, w_{\tau h_{H}}\right)$ lies outside $N_{\tau}\left(\mathfrak{S}_{\tau}\right)$. By Lemma 4.2, we could then take a subsequence converging for $H \rightarrow 0$ in the weak* topology indicated in (4.14) to some limit lying in $\mathfrak{S}_{\tau}$. As this topology is finer than the metrizable topology considered so far, this subsequence would converge in this coarser topology and eventually (i.e. for $H$ small enough) would lie in $B_{\rho}(0)$ or, more precisely, in the compact set $B_{\rho}(0) \backslash N_{\tau}\left(\mathfrak{S}_{\tau}\right)$, which would show that this limit is simultaneously in $B_{\rho}(0) \backslash N_{\tau}\left(\mathfrak{S}_{\tau}\right)$ and in $\mathfrak{S}_{\tau}$, which is not possible. 
Merging the obtained inclusions $\mathfrak{S}_{\tau h} \subset N_{\tau}\left(\mathfrak{S}_{\tau}\right)$ and $\mathfrak{S}_{\tau} \subset N_{\epsilon}\left(\mathfrak{S}_{0}\right)$, we can deduce $\mathfrak{S}_{\tau h} \subset N_{\tau}\left(\mathfrak{S}_{\tau}\right) \subset$ $N_{\tau}\left(N_{\epsilon}\left(\mathfrak{S}_{0}\right)\right)=N_{\tau+\epsilon}\left(\mathfrak{S}_{0}\right) \subset N_{\tau_{\epsilon}+\epsilon}\left(\mathfrak{S}_{0}\right) \subset N_{2 \epsilon}\left(\mathfrak{S}_{0}\right)$. Altogether, we thus have shown that for this $H(\tau)$ and for any $0<h \leq H(\tau)$, any discrete solution $\left(u_{\tau h}, \pi_{\tau h}, \eta_{\tau h}, w_{\tau h}\right) \in \mathfrak{S}_{\tau h} \subset N_{\tau_{\epsilon}+\epsilon}\left(\mathfrak{S}_{0}\right)$, and that this holds for any $\tau \leq \tau_{\epsilon}$. As we can push $\epsilon \rightarrow 0$ (and also $\tau_{\epsilon} \rightarrow 0$ ), we verify the convergence claimed in (ii).

Remark 4.9. The assertion of Corollary 4.8 involves only implicitly stated a stability criterion $h \leq H(\tau)$ and is, unfortunately, of limited practical use in actual calculations. A more explicit form of $H$ seems difficult to obtain. It might expectedly require some regularity, but this is not easy to prove even for the isothermal plasticity. E.g. recent results from [18] require the loading in $L^{\infty}\left(I ; L^{2}\left(\Omega ; \mathbb{R}^{d}\right)\right)$, but in the anisothermal case the loading by $\nabla \theta, c f .(2.10 \mathrm{a})$, is here a-priori in $L^{r}\left(Q ; \mathbb{R}^{d}\right)$ only, $c f .(4.27 \mathrm{~d})$. Therefore, sophisticated bootstrap arguments or joint regularity estimates like [14], Section 5.4 (where no plasticity is involved, however), would be necessary. All this obviously exceed the scope of the presented exposition, however.

\section{Computational implementation and 3D simulations}

In our implementation we made the simplification $\mathcal{S}=0$ and solved the variational inclusion exactly, making use of the fact that $\omega_{\tau h}^{k} \in \partial \delta_{K}^{*}\left(d_{t} z_{\tau h}^{k}\right)$ holds if and only if $d_{t} z_{\tau h}^{k} \in \partial \delta_{K}\left(\omega_{\tau h}^{k}\right)$, where $z_{\tau h}^{k}=\left(\pi_{\tau h}^{k}, \eta_{\tau h}^{k}\right)$ and $\omega_{\tau h}^{k}=\left(\widetilde{\sigma}_{\tau h}^{k}, \xi_{\tau h}^{k}\right)=\left(\mathbb{C}\left(e\left(u_{\tau h}^{k}\right)-\pi_{\tau h}^{k}\right)-\mathbb{H} \pi_{\tau h}^{k},-b \eta_{\tau h}^{k}\right)$. For our numerical simulation, we neglected the kinematic hardening by putting $\mathbb{H}=0$. We introduce $A_{\tau h}^{k}:=d_{t} e\left(u_{\tau h}^{k}\right)-\tau^{-1} \mathbb{C}^{-1} \widetilde{\sigma}_{\tau h}^{k-1}$ and use the identity $\pi_{\tau h}^{k}=$ $e\left(u_{\tau h}^{k}\right)-\mathbb{C}^{-1} \omega_{\tau h}^{k}$ to recast the flow rule as

$$
\left(A_{\tau h}^{k}-\tau^{-1} \mathbb{C}^{-1} \widetilde{\sigma}_{\tau h}^{k}, d_{t} \eta_{\tau h}^{k}\right) \in \partial \delta_{K}\left(\widetilde{\sigma}_{\tau h}^{k}, \xi_{\tau h}^{k}\right)
$$

For certain material laws and stress-strain relations it is possible to derive an explicit formula for the unique solution $\tilde{\sigma}_{\tau h}^{k}, \eta_{\tau h}^{k}$ of (5.1) in terms of (given) $A_{\tau h}^{k}, \xi_{\tau h}^{k-1}$, and $\tau$. As above, we employ the linear stress-strain relation $\tilde{\sigma}_{\tau h}^{k}=\mathbb{C} \varepsilon_{\tau h}^{k}=\lambda_{\mathrm{e}} \operatorname{tr} \varepsilon_{\tau h}^{k} \mathbb{I}+2 \mu_{\mathrm{e}} \varepsilon_{\tau h}^{k}$ for the elastic strain tensor $\varepsilon_{\tau h}^{k}=e\left(u_{\tau h}^{k}\right)-\pi_{\tau h}^{k}$. We consider mere isotropic hardening defined through the von-Mises yield function $\Phi(\widetilde{\sigma}, \xi):=|\operatorname{dev} \widetilde{\sigma}|-\widetilde{\sigma}_{y}\left(1+q_{H} \xi\right)$ and the corresponding set of admissible pairs of elastic stresses and driving forces for hardening

$$
K:=\left\{(\widetilde{\sigma}, \xi) \in \mathbb{R}_{\mathrm{sym}}^{d \times d} \times \mathbb{R} ;|\operatorname{dev} \widetilde{\sigma}| \leq \widetilde{\sigma}_{y}\left(1+q_{H} \xi\right)\right\},
$$

where $\widetilde{\sigma}_{y}$ is the yield stress, $q_{H}$ the hardening parameter, and "dev" denotes the trace free part of a tensor. With these definitions we are in the setting of [11], Theorem 3.2, and may deduce that for given $A_{\tau h}^{k}, \xi_{\tau h}^{k-1}$, and $\tau>0$ there exists a unique solution $\left(\widetilde{\sigma}_{\tau h}^{k}, \eta_{\tau h}^{k}\right)$ of $(5.1)$ given by

$$
\tilde{\sigma}_{\tau h}^{k}=\Sigma\left(A_{\tau h}^{k}, \xi_{\tau h}^{k-1}, \tau\right):=\left(\lambda_{\mathrm{e}}+2 \mu_{\mathrm{e}} / d\right) \operatorname{tr}\left(\tau A_{\tau h}^{k}\right) \mathbb{I}+F\left(A_{\tau h}^{k}, \xi_{\tau h}^{k-1}, \tau\right) \operatorname{dev}\left(\tau A_{\tau h}^{k}\right)
$$

where

$$
F\left(A_{\tau h}^{k}, \xi_{\tau h}^{k-1}, \tau\right)= \begin{cases}\frac{\tilde{\sigma}_{y}}{\left(1+b q_{H}^{2} \widetilde{\sigma}_{y}^{2}\right)}\left(\frac{\left(1+q_{H} \xi_{\tau h}^{k-1}\right)}{\operatorname{ldev}\left(\tau A_{\tau h}^{k}\right) \mid}+b q_{H}^{2} \widetilde{\sigma}_{y}\right) & \text { for }\left|\operatorname{dev}\left(\tau A_{\tau h}^{k}\right)\right| \geq \frac{\tilde{\sigma}_{y}\left(1+q_{H} \xi_{\tau h}^{k-1}\right)}{2 \mu_{\mathrm{e}}} \\ 2 \mu & \text { for }\left|\operatorname{dev}\left(\tau A_{\tau h}^{k}\right)\right| \leq \frac{\tilde{\sigma}_{y}\left(1+q_{H} \xi_{\tau h}^{k-1}\right)}{2 \mu_{\mathrm{e}}}\end{cases}
$$

and

$$
\xi_{\tau h}^{k}= \begin{cases}\frac{1}{q_{H} \widetilde{\sigma}_{y}}\left(\left|\operatorname{dev} \widetilde{\sigma}_{\tau h}^{k}\right|-\widetilde{\sigma}_{y}\right) & \text { for }\left|\operatorname{dev}\left(\tau A_{\tau h}^{k}\right)\right| \geq \frac{\widetilde{\sigma}_{y}\left(1+q_{H} \xi_{\tau h}^{k-1}\right)}{2 \mu_{\mathrm{e}}} \\ \xi_{\tau h}^{k-1} & \text { for }\left|\operatorname{dev}\left(\tau A_{\tau h}^{k}\right)\right|<\frac{\widetilde{\sigma}_{y}\left(1+q_{H} \xi_{\tau h}^{k-1}\right)}{2 \mu_{\mathrm{e}}}\end{cases}
$$

and $\eta_{\tau h}^{k}=-b^{-1} \xi_{\tau h}^{k}$. In particular, the plastic phase occurs for $\left|\operatorname{dev}\left(\tau A_{\tau h}^{k}\right)\right| \geq \widetilde{\sigma}_{y}\left(1+q_{H} \xi_{\tau h}^{k-1}\right) /\left(2 \mu_{\mathrm{e}}\right)$. For explicit formulas in case of other plastic material behavior such as plasticity with linear kinematic hardening we refer the reader to [11]. 
In addition to the simplifications $\mathcal{S}=0$ and $\mathbb{H}=0$, we neglect inertial and viscous effects, kinematic hardening, and temperature dependence of the heat capacity. Thus, in the numerical experiments reported below, we consider $c_{\mathrm{v}}>0$ constant and set $\varrho:=0$ and $\mathbb{D}:=0$. The discrete scheme $(4.2 \mathrm{a})-(4.2 \mathrm{c})$ then reduces to the following coupled quasi-stationary, displacement and temperature formulation: Given $\left(u_{\tau h}^{k-1}, \xi_{\tau h}^{k-1}, \omega_{\tau h}^{k-1}, \theta_{\tau h}^{k-1}\right) \in$ $V_{1, h}^{d} \times V_{0, h} \times V_{0, h}^{d \times d} \times V_{1, h}$ find $\left(u_{\tau h}^{k}, \theta_{\tau h}^{k}\right) \in V_{1, h}^{d} \times V_{1, h}$ such that $\left.u_{\tau h}^{k}\right|_{\Gamma}=u_{\mathrm{D}, \tau h_{1}}$ and

$$
\begin{aligned}
\int_{\Omega} \Sigma\left(A_{\tau h}^{k}\left[u_{\tau h}^{k}\right], \xi_{\tau h}^{k-1}, \tau\right): e(v) \mathrm{d} x & =\int_{\Omega} \mathbb{C E} \theta_{\tau h}^{k}: e(v) \mathrm{d} x \\
c_{\mathrm{V}}\left(d_{t} \theta_{\tau h}^{k}, w\right)+\int_{\Omega} \mathbb{K} \nabla \theta_{\tau h}^{k} \cdot \nabla w \mathrm{~d} x & =\int_{\Omega} \widetilde{\sigma}_{\tau h}^{k}: d_{t} \pi_{\tau h}^{k} w \mathrm{~d} x-b^{-1} \int_{\Omega} \xi_{\tau h}^{k} d_{t} \xi_{\tau h}^{k} w \mathrm{~d} x
\end{aligned}
$$

for all $v \in V_{1, h}^{d}$ with $\left.v\right|_{\Gamma}=0$ and all $w \in V_{1, h}$.

The implementation of the approximation scheme was done in MATLAB in the spirit of $[2,11]$ and equations (5.4)-(5.5) were decoupled and solved with a fixed-point iteration. In this implementation, the nonlinear system of equations (5.4) is approximated with a Newton iteration and all occurring systems of linear equations are solved using MATLAB's backslash operator. In our experiments the Newton scheme always terminated within at most four iterations to achieve an $\ell^{2}$ norm of the residual vector (defined through nodal basis functions) less than $10^{-7} \mathrm{~J}$. Moreover, in all time steps, less than six fixed point iterations were sufficient to achieve an absolute change of the temperature in the $H^{1}$ norm less than $10^{-6} \mathrm{~km}^{1 / 2}$.

We used the scheme (5.4)-(5.5) to simulate the plastification through thermal expansion of a steel cubicshaped specimen subject to an external heating, starting from room temperature and without initial plastic strain. Focusing on this process, we neglect surface loading, i.e. $g=0$. To demonstrate interesting ratedependence of the whole system, we considered different speeds of the heating regime but with the same total energy pumped into the specimen. This is specified in the following example:

- Material data: heat capacity $c_{\mathrm{v}}=3.2 \mathrm{MJ} \cdot \mathrm{m}^{-3} \cdot \mathrm{K}^{-1}$, heat transfer coefficient $\kappa=80 \mathrm{~W} \cdot \mathrm{m}^{-1} \cdot \mathrm{K}^{-1}$, thermal-expansion coefficient $\alpha=2 \times 10^{-5} \mathrm{~K}^{-1}$, the Young's modulus $E=137 \mathrm{GPa}$, the Poisson ratio $\nu=0.3$. The set of admissible stresses is defined through $\widetilde{\sigma}_{y}:=450 \mathrm{MPa}$ and $q_{H}=10^{-3} \mathrm{~Pa}^{-1}$. The plastic part of the free energy is defined through the parameter $b=10^{-3} \mathrm{~Pa}$.

- Geometry of the specimen: $d:=3, \Omega:=(-L / 2, L / 2)^{3}$ for $L=2 \times 10^{-2} \mathrm{~m}$.

- Initial conditions: $u_{0}(x)=\alpha \theta_{0} x$ for $x \in \Omega, \pi_{0}:=0, \eta_{0}:=0$, and $\theta_{0}=300 \mathrm{~K}$.

- Heating regime: considering $T=1.5 \mathrm{~s}$ and given $t_{*} \in[0, T]$, we put the heat flux

$$
f(t, x):= \begin{cases}t_{*}^{-1} \cdot 10^{6} \mathrm{~J} \cdot \mathrm{m}^{-2} & \text { for } t<t_{*} \\ 0 & \text { for } t \geq t_{*}\end{cases}
$$

for $t \in[0, T]$ and $x \in \Gamma$.

Let us remark that the Lamé constants used in (2.19) are calculated, as standard, $\lambda_{\mathrm{e}}=\nu E /((1+\nu)(1-2 \nu))$ and $\mu_{\mathrm{e}}=E /(2(1+\nu))$. The value of the heat capacity $c_{\mathrm{v}}$ corresponds to the capacity per mass $400 \mathrm{~J} \cdot \mathrm{kg}^{-1} \cdot \mathrm{K}^{-1}$ if the mass density of the conventional steel $8 \times 10^{3} \mathrm{~kg} \cdot \mathrm{m}^{-3}$ is considered. The overall energy pumped into the body $\int_{0}^{T} \int_{\Gamma} f \mathrm{~d} S \mathrm{~d} t=\int_{0}^{t_{*}} \int_{\Gamma} f \mathrm{~d} S \mathrm{~d} t=10^{6} \mathrm{~J} \cdot \mathrm{m}^{-2} \operatorname{meas}_{2}(\Gamma)$ is thus $2400 \mathrm{~J}$ independently of $t^{*}$.

We simplify computationally this model problem by exploiting the symmetry of data, i.e. both of geometry and of the initial conditions as well as of the heating sources, and restricting to the subdomain $\Omega^{\prime}:=(0, L / 2)^{3}$. This enforces us to implement gliding boundary conditions along the three sides $S_{i}:=\left\{\left(x_{1}, x_{2}, x_{3}\right) \in \bar{\Omega}^{\prime} ; x_{i}=0\right\}$ with $i=1,2,3$, i.e., to impose (homogeneous) Dirichlet conditions on $u_{i}$ on $S_{i}$ and a (homogeneous) Neumann condition on the remaining components of $u$ as well as on $w$. Thus we pre-select only some symmetrical solutions of the original problem on $\Omega$. One should realize that, due to lack of rigorous uniqueness proof, only the whole set of solutions must be symmetric and non-symmetric solutions may exist. Anyhow, this set contains also some symmetric solutions, which can be proved just by applying the previous arguments to the problem reduced on $\Omega^{\prime}$. 
For a triangulation of $\Omega^{\prime}$ into 2560 tetrahedra obtained from three uniform refinements of a coarse triangulation of $\Omega^{\prime}$ into 5 tetrahedra (i.e. $h=2^{-3} \sqrt{3} L / 2 \approx 0.2 \times 10^{-2} \mathrm{~m}$ ) and used for both equations (5.4) and (5.5), we employed the time-step size $\tau=v h$ with $v=0.05 \mathrm{~m} \cdot \mathrm{s}^{-1}$. Figure 1 illustrates the evolution defined through $t_{*}=0.075 \mathrm{~s}$. The heat energy is pumped through the sides of the body, which leads to higher temperatures along the sides and especially the edges and the corners. This non-uniform temperature (and thus thermal expansion) distribution enforces elastic stresses which are large along the edges of $\Omega$ and cause an expansion of the body. At $t \approx 0.035 \mathrm{~s}$ the stresses attain the yield stress in vicinity of the edges and trigger plastic strain evolution. In contrast with it, there is no plastic strain around the free corners (only one of which is depicted in Figs. 1 and 2 due to the smaller computational domain $\Omega^{\prime} \varsubsetneqq \Omega$ ), which is is due to the fact that the deformation is there locally a compression and no shear forces occur. When the external heat flux $f$ is switched off at $t_{*}=0.075 \mathrm{~s}$, the average temperature in $\Omega^{\prime}$ no longer increases and the temperature equidistributes after some time. In contrast, the stresses cannot equidistribute and the specimen cannot entirely return towards its initial stress-free state if plasticized at some regions (here along edges) during the fast heating process. Figure 1 (middle bottom snapshot) indeed shows remaining elastic stress especially in the central region of the specimen.

Figure 2 illustrates, in particular, that for decreasing mesh-sizes the plastic strain becomes more and more symmetric. The asymmetry on coarse meshes is expectedly due to the anisotropy of the underlying triangulation, although, due to lack of rigorous uniqueness proof, only the whole set of solutions to the limit problem $(i . e . h=0)$ must be symmetric and non-symmetric solutions may exist, and we thus even cannot claim that the concrete approximate solutions approximate any symmetric solution and exhibit some tendency for symmetry.

In Figure 3 we graphically studied the validity of a discrete energy balance analogous to the continuous one in (3.7d). The left plot of Figure 3 shows the total discrete energy $E_{\text {tot }}^{h}$ and the work of external heat $W_{\text {ext }}^{h}$ plus initial energy $E_{\text {tot }}^{h}(0)$, defined by

$$
E_{\text {tot }}^{h}\left(t_{k}\right):=\int_{\Omega} c_{\mathrm{v}} \theta_{\tau h}^{k}+\frac{1}{2} \mathbb{C}^{-1} \widetilde{\sigma}_{\tau h}^{k}: \sigma_{\tau h}^{k}+b\left|\xi_{\tau h}^{k}\right|^{2} \mathrm{~d} x, \quad W_{\text {ext }}^{h}\left(t_{k}\right):=\sum_{\ell=0}^{k} \tau \int_{\Gamma} g \mathrm{~d} s .
$$

The two quantities almost coincide for all $t \in[0, T]$ and the right plot of Figure 3 shows their relative distance $\delta_{h}$ defined through

$$
\delta_{h}\left(t_{k}\right):=\frac{\left|E_{\mathrm{tot}}^{h}\left(t_{k}\right)-E_{\mathrm{tot}}^{h}\left(t_{0}\right)-W_{\mathrm{ext}}^{h}\left(t_{k}\right)\right|}{\left|E_{\mathrm{tot}}^{h}(0)+W_{\mathrm{ext}}^{h}\left(t_{k}\right)\right|}
$$

for $h \doteq 2^{-\ell} \sqrt{3} \times 10^{-2} \mathrm{~m}, \ell=3,4,5$. We observe that the relative difference is small and decays linearly to zero as the mesh-size becomes small. The increase of the quantities by approximately $300 \mathrm{~J}$ corresponds to an eighth of the total energy pumped into the entire specimen $\Omega$.

In Figure 4 we displayed for different values of $t_{*}$ the $L^{2}$ norms of the stresses $\widetilde{\sigma}_{\tau h}^{k}-\mathbb{C E} P_{h, 0} \theta_{\tau h}^{k}$ and plastic strains $\pi_{\tau h}^{k}$ as functions of $t / t_{*}$. The $L^{2}$ norm of the stresses increases within the relative-time interval $[0,1]$. For small values of $t_{*}$, i.e., for a faster heating of the specimen, the material is plasticized in large domains. Since for slow heating of the specimen, the temperature rather equidistributes and does not lead to large elastic stresses so that no plastification occurs at all, if $t_{*}$ is $0.3 \mathrm{~s}$ or bigger. Finally, in Figure 5 we plotted the thermal part of the energy, i.e., the quantity $c_{\mathrm{v}} \int_{\Omega} \theta_{\tau h}^{k} \mathrm{~d} x$ (i.e., up to a factor $c_{\mathrm{v}}|\Omega|=25.6 \mathrm{~J} \cdot \mathrm{K}^{-1}$, the average temperature) as a function of $t / t_{*}$. We see that the achieved average temperature is slightly lower for more pronounced plastic process, i.e. for faster heating ( $=$ a shorter time $t_{*}$ ) because bigger part of the heat energy pumped into the body is converted into remaining plastic changes of the material and to the elastic stored energy due to the mentioned remaining elastic stress. This effect is, however, relatively very small ( $c f$. Fig. 5 left) because the energetics of mechanical processes is "cheaper" than the thermal energetics, and can only be made visible on some detailed zoom ( $c f$. Fig. 5 right). 

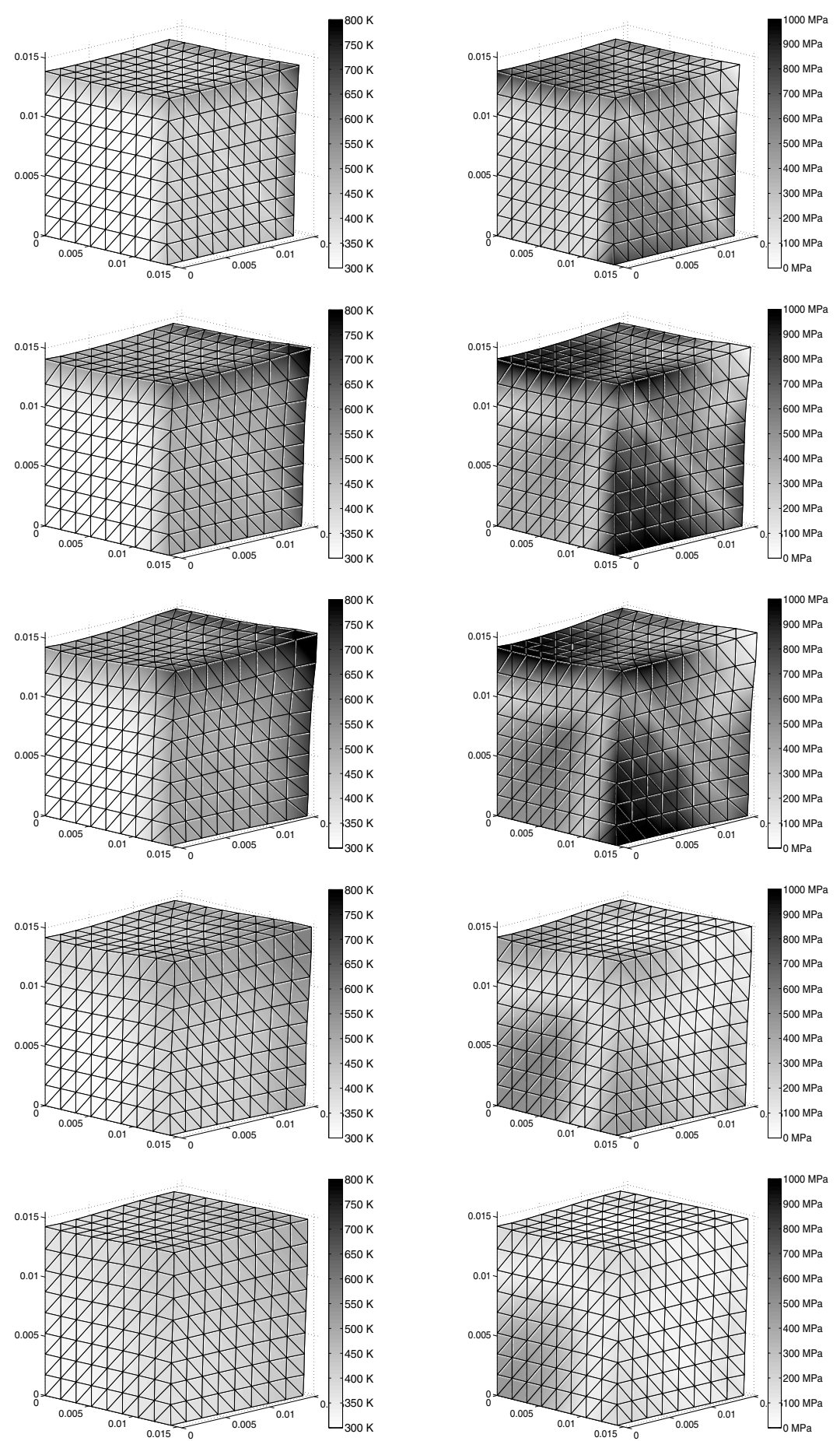
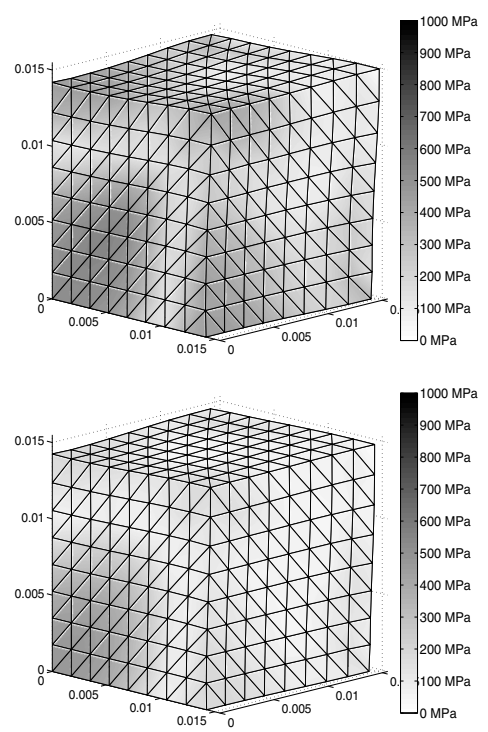
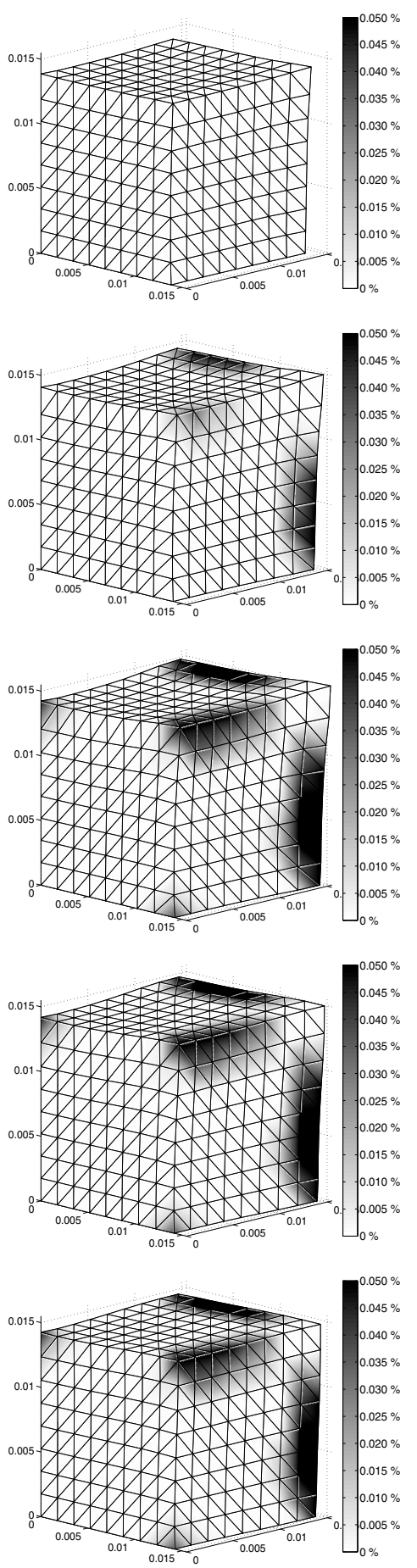

Figure 1. Displacement (magnified by factor 60 ) together with temperature $\theta_{\tau h}(t, \cdot)$, postprocessed modulus of stresses $\left|\sigma_{\tau h}(t, \cdot)\right|$, and postprocessed modulus of plastic strain $\left|\pi_{\tau h}(t, \cdot)\right|$ (from left to right) for $t=0.025,0.05,0.075,0.15,0.3 \mathrm{~s}$ (from top to bottom) with $f$ defined through (5.6) with $t_{*}=0.075 \mathrm{~s}$. 

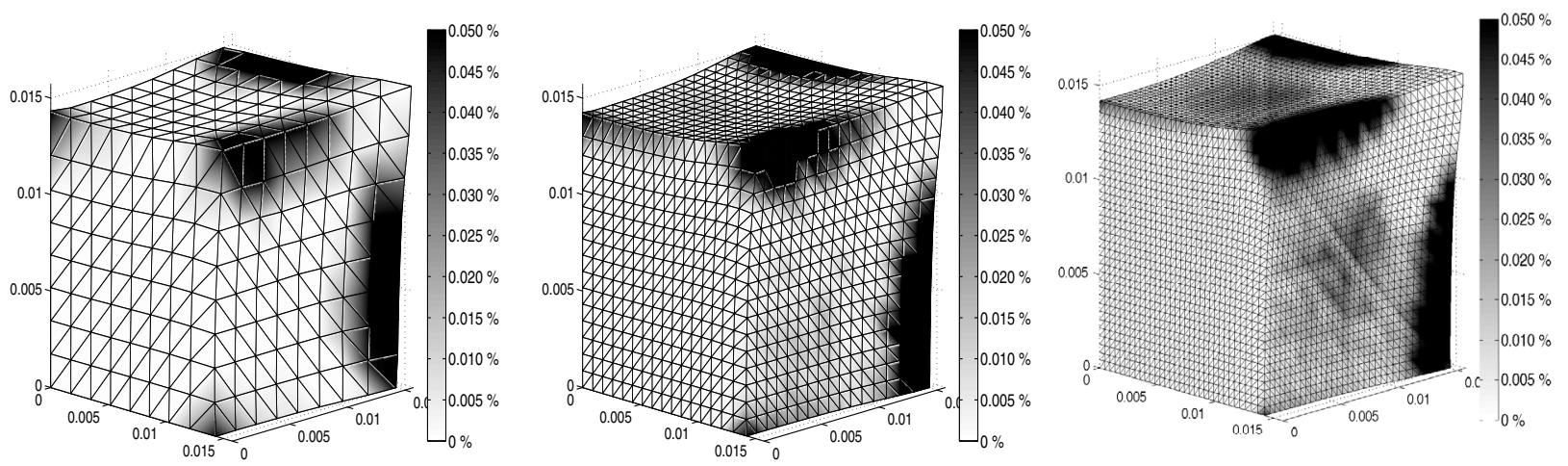

Figure 2. Postprocessed modulus of plastic strain $\left|\pi_{\tau h}(t, \cdot)\right|$ for $t=t_{*}=0.075 \mathrm{~s}$ and the mesh sizes $h=2^{-\ell} \sqrt{3} \times 10^{-2} \mathrm{~m}, \ell=3,4,5$ (from left to right). The finest mesh has 163840 tetrahedral elements.
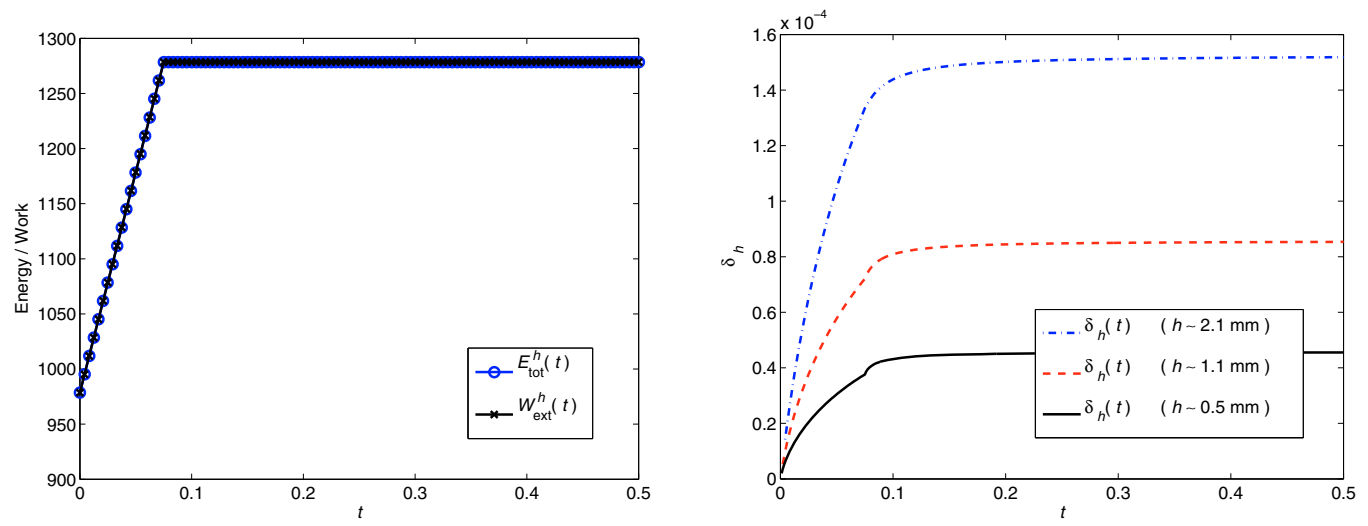

FIGURE 3. Total discrete energy and work of external heat (left). The relative difference between the total discrete energy and the external forces is small and converges linearly to zero as $h \rightarrow 0$ (right).
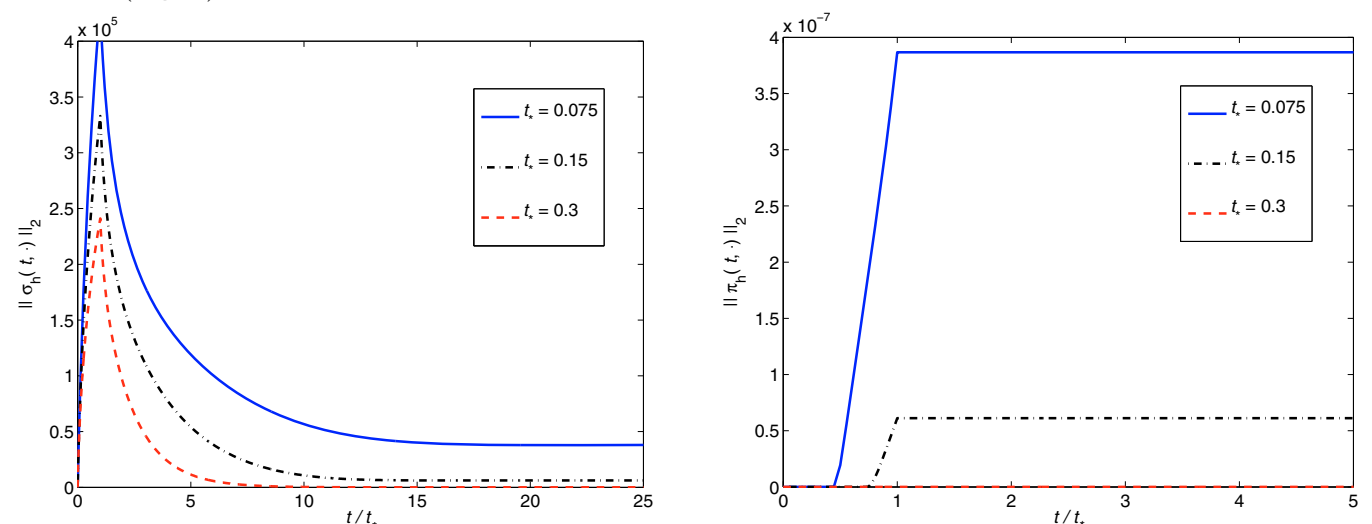

Figure 4. $L^{2}$ norms of discrete stresses (left) and plastic strains (right) as functions of the relative time $t / t_{*}$ for heating times $t_{*}=0.075,0.15,0.3 \mathrm{~s}$. The plastic material behaviour becomes less pronounced as the external heating happens slower. 

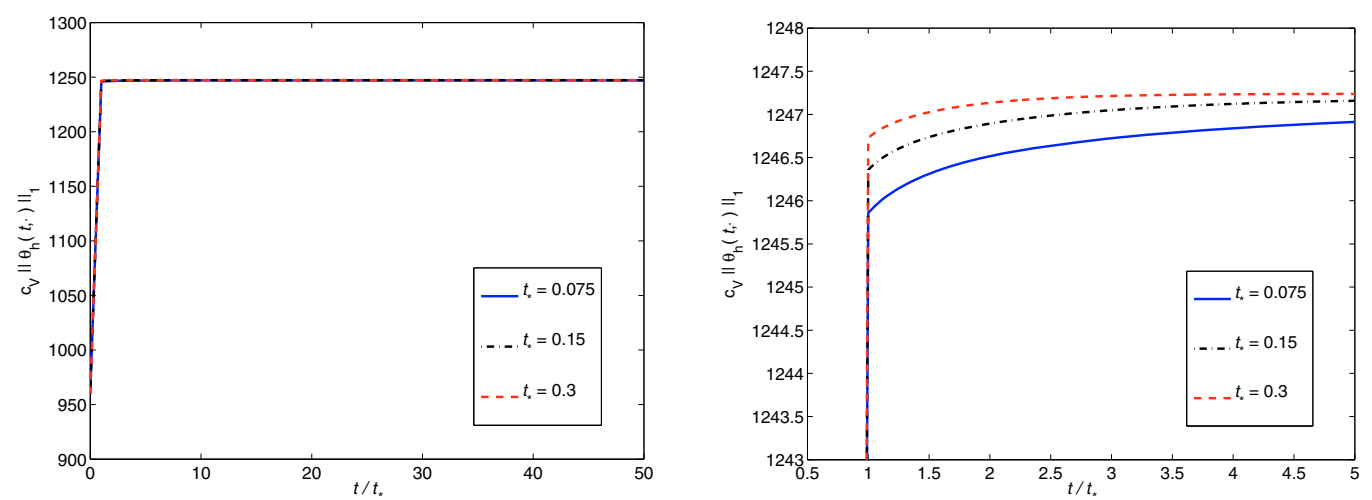

FiguRE 5. Thermal part of the energy for different speed of the heating regimes given by (5.6) for $t_{*}=0.075,0.15,0.3 \mathrm{~s}$. The detailed picture (right) shows that final temperature is slightly lower if the material was more plasticized during the heating.

\section{REFERENCES}

[1] C. Agelet de Saracibar, M. Cervera and M. Chiumenti, On the formulation of coupled thermoplastic problems with phasechange. Int. J. Plasticity 15 (1999) 1-34.

[2] J. Alberty, C. Carstensen and S.A. Funken, Remarks around 50 lines of Matlab: short finite element implementation. Numer. Algorithms 20 (1999) 117-137.

[3] S. Bartels and T. Roubíček, Thermoviscoplasticity at small strains. ZAMM 88 (2008) 735-754.

[4] L. Boccardo, A. Dall'aglio, T. Gallouët and L. Orsina, Nonlinear parabolic equations with measure data. J. Funct. Anal. 147 (1997) 237-258.

[5] L. Boccardo and T. Gallouët, Non-linear elliptic and parabolic equations involving measure data. J. Funct. Anal. 87 (1989) 149-169.

[6] L. Boccardo and T. Gallouët, Summability of the solutions of nonlinear elliptic equations with right hand side measures. J. Convex Anal. 3 (1996) 361-365.

[7] B.A. Boley and J.H. Weiner, Theory of thermal stresses. J. Wiley (1960), Dover edition (1997).

[8] S.C. Brenner and L.R. Scott, The mathematical theory of finite element methods. Springer, second edition, New York (2002).

[9] O. Bruhns and J. Mielniczuk, Zur Theorie der Verzweigungen nicht-isothermer elastoplastischer Deformationen. Ing.-Arch. 46 (1977) 65-74.

[10] M. Canadija and J. Brnic, Associative coupled thermoplasticity at finite strain with temperature-dependent material parameters. Int. J. Plasticity 20 (2004) 1851-1874.

[11] C. Carstensen and R. Klose, Elastoviscoplastic finite element analysis in 100 lines of Matlab. J. Numer. Math. 10 (2002) $157-192$.

[12] G. Dal Maso, G.A. Francfort and R. Toader, Quasistatic crack growth in nonlinear elasticity. Arch. Rational Mech. Anal. 176 (2005) 165-225.

[13] G. Dal Maso, A. DeSimone and M.G. Mora, Quasistatic evolution problems for linearly elastic-perfectly plastic materials. Arch. Ration. Mech. Anal. 180 (2006) 237-291.

[14] C. Eck, J. Jarušek and M. Krbec, Unilateral Contact Problems. Chapman \& Hall/CRC, Boca Raton (2005).

[15] G. Francfort and A. Mielke, An existence result for a rate-independent material model in the case of nonconvex energies. J. reine angew. Math. 595 (2006) 55-91.

[16] P. Hakansson, M. Wallin and M. Ristinmaa, Comparison of isotropic hardening and kinematic hardening in thermoplasticity. Int. J. Plasticity 21 (2005) 1435-1460.

[17] S. Hu and N.S. Papageorgiou, Handbook of Multivalued Analysis. Kluwer, Dordrecht, Part I (1997), Part II (2000).

[18] D. Knees, On global spatial regularity and convergence rates for time dependent elasto-plasticity. Math. Models Methods Appl. Sci. (2010) DOI: 10.1142/S0218202510004805.

[19] G.A. Maughin, The Thermomechanics of Plasticity and Fracture. Cambridge Univ. Press, Cambridge (1992).

[20] C. Miehe, A theory of large-strain isotropic thermoplasticity based on metric transformation tensor. Archive Appl. Mech. 66 (1995) 45-64.

[21] A. Mielke, Evolution of rate-independent systems, in Handbook of Differential Equations: Evolut. Diff. Eqs., C. Dafermos and E. Feireisl Eds., Elsevier, Amsterdam (2005) 461-559. 
[22] A. Mielke and T. Roubíček, Numerical approaches to rate-independent processes and applications in inelasticity. ESAIM: M2AN 43 (2009) 399-428.

[23] A. Mielke and and F. Theil, A mathematical model for rate-independent phase transformations with hysteresis, in Models of continuum mechanics in analysis and engineering, H.-D. Alber, R. Balean and R. Farwing Eds., Shaker Ver., Aachen (1999) $117-129$.

[24] A. Mielke and F. Theil, On rate-independent hysteresis models. Nonlin. Diff. Eq. Appl. 11 (2004) $151-189$.

[25] A. Mielke, T. Roubíček and U. Stefanelli, $\Gamma$-limits and relaxations for rate-independent evolutionary problems. Calc. Var. PDE 31 (2008) 387-416.

[26] T.D.W. Nicholson, Large deformation theory of coupled thermoplasticity including kinematic hardening. Acta Mech. 142 (2000) 207-222.

[27] P. Rosakis, A.J. Rosakis, G. Ravichandran and J. Hodowany, A thermodynamic internal variable model for the partition of plastic work into heat and stored energy in metals. J. Mech. Phys. Solids 48 (2000) 581-607.

[28] T. Roubíček, Nonlinear Partial Differential Equations with Applications. Birkhäuser, Basel (2005).

[29] T. Roubíček, Thermo-visco-elasticity at small strains with $L^{1}$-data. Quart. Appl. Math. 67 (2009) 47-71.

[30] T. Roubíček, Rate independent processes in viscous solids at small strains. Math. Methods Appl. Sci. 32 (2009) 825-862.

[31] T. Roubíček, Thermodynamics of rate independent processes in viscous solids at small strains. SIAM J. Math. Anal. 42 (2010) $256-297$.

[32] A. Srikanth and N. Zabaras, A computational model for the finite element analysis of thermoplasticity coupled with ductile damage at fonite strains. Int. J. Numer. Methods Eng. 45 (1999) 1569-1605.

[33] Q. Yang, L. Stainier and M. Ortiz, A variational formulation of the coupled thermo-mechanical boundary-value problem for general dissipative solids. J. Mech. Phys. Solids 54 (2006) 401-424.

[34] H. Ziegler, A modification of Prager's hardening rule. Quart. Appl. Math. 17 (1959) 55-65. 